\title{
Imaging transient species in the femtosecond $A$-band photodissociation of $\mathrm{CH}_{3} \mathrm{I}$
}

\author{
J. Durá, ${ }^{1,2}$ R. de Nalda, ${ }^{3}$ G. A. Amaral, ${ }^{1}$ and L. Bañares ${ }^{1, a)}$ \\ ${ }^{1}$ Departamento de Química Física I, Facultad de Ciencias Químicas, Universidad Complutense de Madrid, \\ 28040 Madrid, Spain \\ ${ }^{2}$ Instituto de Estructura de la Materia, CSIC, C/Serrano 123, 28006 Madrid, Spain \\ ${ }^{3}$ Instituto de Química Física Rocasolano, CSIC, C/Serrano 119, 28006 Madrid, Spain
}

(Received 13 July 2009; accepted 3 September 2009; published online 7 October 2009)

\begin{abstract}
A nonresonant femtosecond laser pulse centered at $802 \mathrm{~nm}$ is used to probe the real time photodissociation dynamics of $\mathrm{CH}_{3} \mathrm{I}$ in the $A$-band at $267 \mathrm{~nm}$. Using multiphoton ionization with this probe laser pulse and velocity map ion imaging of $\mathrm{CH}_{3}{ }^{+}$, we have followed the time evolution of the translational energy and spatial anisotropy of the $\mathrm{CH}_{3}$ fragment, which in turn has permitted to image the $\mathrm{C}-\mathrm{I}$ bond breaking from the initial Franck-Condon region up to the final products along the reaction coordinate. Given the temporal width of our pump and probe laser pulses ( $\sim 80 \mathrm{fs})$, a mechanism is proposed by which transient species are probed by simultaneous absorption of pump and probe laser pulses through intermediate Rydberg and ionic states of $\mathrm{CH}_{3} \mathrm{I}$ while the pump and probe pulses overlap in time. This study shows how the combination of femtosecond multiphoton ionization and ion imaging techniques provides an ideal tool to resolve in time the different stages of the bond breaking event in a polyatomic molecule. (C) 2009 American Institute of Physics. [doi:10.1063/1.3236808]
\end{abstract}

\section{INTRODUCTION}

From the early days of reaction dynamics, one of the main aims in the field has been the detection and characterization of transition states in chemical reactions. ${ }^{1}$ In the pioneering femtosecond time-resolved experiments carried out by Zewail and co-workers ${ }^{2}$ in the late 1980s, the real time photodissociation dynamics of ICN was resolved by tuning the probe laser wavelength on-resonance to the first electronic excited state of the free $\mathrm{CN}$ fragment, which then fluoresces. In this case, the resonant probe laser opens up an optical coupling region in the potential energy surface determined by its bandwidth, which allows the clocking of the reaction from the initial Franck-Condon wave packet to the free fragments in the asymptotic region. By slightly detuning the probe laser wavelength, however, inner parts of the potential surface can be accessed and transient species along the reaction coordinate can be detected. ${ }^{2}$

In more recent years, Zewail and co-workers ${ }^{3}$ introduced the femtosecond time-resolved kinetic energy time-of-flight spectroscopy to study elementary steps of complex reactions. ${ }^{4}$ Using this technique, they were able to follow the dynamics of final products and transient species in just one experiment by resolving the kinetic energy of a given fragment as a function of the delay time between the pump and probe laser pulses and using only one wavelength for the probe step. In a sense, this approach is analogous to the anion femtosecond photoelectron spectroscopy introduced by Greenblatt et al., ${ }^{5}$ in which a first femtosecond laser pulse excites a ground-state anion to a repulsive potential energy surface and a second, time-delayed, femtosecond laser pulse

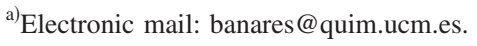

is used to detach the electron from the anion. The dynamics of the anion is followed by the evolution of the resulting different kinetic energy electrons when the wave packet is mapped onto the accessible neutral potential surfaces.

More recently, Strasser et al. ${ }^{6}$ combined time-resolved photoelectron spectroscopy and ultrafast extreme ultraviolet (XUV) light at $53.7 \mathrm{~nm}$ obtained by high order harmonic generation to probe the photodissociation of $\mathrm{Br}_{2}\left({ }^{1} \Pi_{u}\right)$ by one-photon ionization. The XUV pulse allows the simultaneous probe of all stages of dissociation, starting from the measurement of ground state depletion, followed by measurements of a photoelectron spectrum of the wave packet excited on the $\operatorname{Br}_{2}\left({ }^{1} \Pi_{u}\right)$ state, detection of nearly separated $\mathrm{Br}$ atom pairs, and fully separated $\mathrm{Br}$ atoms. The measured transient binding energy shifts in $\mathrm{Br}$ photoelectron peaks were interpreted as due to photoionization of nearly separated atom pairs onto dissociative ionization states. It was argued that the advantage of using XUV light as a probe source is that it allows single-photon ionization of valence and core electrons from time-evolving excited states as well as the ground state system. In contrast to multiphoton ionization (MPI), which typically allows the probing of the excited state only in a detection window that has a favorable ionization probability, XUV photoionization is not limited to a specific configuration of the excited state, making it a more general probe of dynamics.

In order to get similar dynamics of transient species and final products, the strategy followed in the present work has been to use MPI with a very off-resonant probe laser pulse centered at $802 \mathrm{~nm}$. Actually, for the $\mathrm{CH}_{3} \mathrm{I}$ molecule under study, at least seven photons of $802 \mathrm{~nm}$ are needed to ionize $\mathrm{CH}_{3} \mathrm{I}$ and the $\mathrm{CH}_{3}$ and I fragments. In contrast with the resonant experiment, where the probing of the fragment only 
starts when the system has evolved long enough along the reaction coordinate (optical coupling region), the very offresonant experiment would allow the detection of free fragments and transient species. The additional ingredient of our strategy is the use of the velocity map imaging technique. This technique permits full three-dimensional (3D) spatial resolution of the velocity distribution of fragments, which, coupled to femtosecond pump-probe detection, leads to a complete real time elucidation of the dissociation event. Using this imaging technique and resonance enhanced multiphoton ionization (REMPI) detection of the $\mathrm{CH}_{3}$ fragment, we have been able to clock the multichannel photodissociation of $\mathrm{CH}_{3} \mathrm{I}$ in the $A$-band. ${ }^{7,8}$ In those works, we measured the reaction times yielding $\mathrm{I}\left({ }^{2} P_{3 / 2}\right)$ and $\mathrm{I}^{*}\left({ }^{2} P_{1 / 2}\right)$ fragments in correlation with vibrational energy channeled into the $\mathrm{CH}_{3}$ cofragment, either on the $\mathrm{C}-\mathrm{H}$ symmetric stretch $\left(\nu_{1}\right)$ or the umbrella mode $\left(\nu_{2}\right){ }^{8}$ The results obtained have posed a challenge for wave packet calculations on the available potential energy surfaces, since reduced dimensionality models have been shown to be unable to describe, even qualitatively, some of the observations. ${ }^{8}$

$\mathrm{CH}_{3} \mathrm{I}$ has been considered a benchmark for photodissociation studies of polyatomic molecules where experimental and theoretical techniques have been tested for more than two decades (see Refs. 8 and 9 for a review). This molecule presents a relatively low number of atoms and an apparently simple dissociation mechanism mediated by a nonadiabatic transition. Most of the studies have focused on the near UV photodissociation via excitation in the first absorption band (A-band), a broad featureless absorption continuum (220-350 nm) with a maximum at $\sim 260 \mathrm{~nm}$. The lowest energy electronic excitation corresponds to a $n \rightarrow \sigma^{*}$ transition, where a nonbonding $p$ electron of iodine is promoted to the lowest energy antibonding molecular orbital available. Due to the strong spin-orbit coupling, the electronic configuration gives rise to five states: $E, E, A_{1}, A_{2}$, and $E$ for $\Omega$ $=2,1,0+, 0-$, and 1, respectively. ${ }^{10}$ In Mulliken ${ }^{11}$ notation, these five states are denoted as ${ }^{3} Q_{2},{ }^{3} Q_{1},{ }^{3} Q_{0^{+}},{ }^{3} Q_{0^{-}}$, and ${ }^{1} Q_{1}$, of which only the ${ }^{3} Q_{1},{ }^{3} Q_{0^{+}}$, and ${ }^{1} Q_{1}$ states can be accessed by dipole allowed transitions. The first and third ones correspond to perpendicular transitions, whereas the second one corresponds to a parallel transition. Upon excitation to the $A$-band, the molecule promptly dissociates via two different channels (in $C_{3 v}$ point group), ${ }^{12}$

$$
\begin{aligned}
\mathrm{CH}_{3} \mathrm{I}\left(\tilde{X}^{1} A_{1}\right)+h \nu & \rightarrow \mathrm{CH}_{3}\left(\tilde{X}^{2} A_{2}^{\prime \prime}\right)+\mathrm{I}\left({ }^{2} P_{3 / 2}\right), \\
& \rightarrow \mathrm{CH}_{3}\left(\tilde{X}^{2} A_{2}^{\prime \prime}\right)+\mathrm{I}^{*}\left({ }^{2} P_{1 / 2}\right),
\end{aligned}
$$

where dissociation energy $D_{0}=2.41 \mathrm{eV}$ for channel (1) and channel (2) is $0.943 \mathrm{eV}$ above that asymptote, which corresponds to the spin-orbit splitting of $\mathrm{I}\left({ }^{2} P\right)$. It is well known that the final branching ratio $\mathrm{I}^{*} /\left(\mathrm{I}+\mathrm{I}^{*}\right)$ depends strongly on the photodissociation wavelength, varying from 0.9 at 267 $\mathrm{nm}$ to almost 1 at $280 \mathrm{~nm}$ and decreasing dramatically afterward to as low as 0.2 at $310 \mathrm{~nm} .{ }^{12}$ Only the ${ }^{3} Q_{0^{+}}$state correlates adiabatically to channel (2), which in addition to the strong parallel anisotropy observed in the $\mathrm{CH}_{3}$ signal arising for both channels (1) and (2), suggests that in the maximum

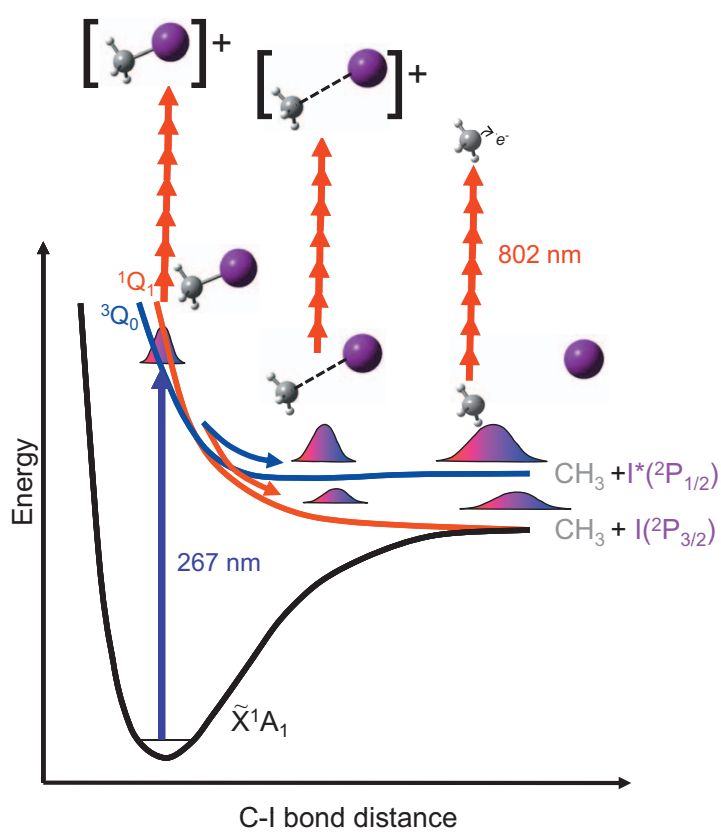

FIG. 1. Schematic representation of the relevant potential energy surfaces along the $\mathrm{C}-\mathrm{I}$ bond representing the $A$-band photodissociation of $\mathrm{CH}_{3} \mathrm{I}$. A nuclear wave packet initially prepared in the ${ }^{3} Q_{0}$ potential energy curve by a $267 \mathrm{~nm}$ femtosecond laser pulse evolves adiabatically on that surface yielding $\mathrm{CH}_{3}+\mathrm{I}^{*}\left({ }^{2} P_{1 / 2}\right)$. A portion of the initial wave packet leaks nonadiabatically to the ${ }^{1} Q_{1}$ surface, which correlates with $\mathrm{CH}_{3}+\mathrm{I}\left({ }^{2} P_{3 / 2}\right)$. The main aim of the present study is to follow the real time dynamics of the photodissociation process from reagents to products through transient species by probing with nonresonant MPI using an IR probe pulse $(802 \mathrm{~nm})$.

of the $A$-band most of the absorption occurs into the ${ }^{3} Q_{0^{+}}$ state and that a small fraction of the initial wave packet is able to leak by nonadiabatic crossing to the ${ }^{1} Q_{1}$ potential energy surface to produce the observed small amount of products from channel (1). In general terms, the accepted dissociation mechanism for $\mathrm{CH}_{3} \mathrm{I}$ in the $A$-band is that of a direct, prompt bond breakage, in which a nonadiabatic crossing between surfaces is involved.

As mentioned above, in an attempt to observe transient dynamics at the early stages of the dissociation in the $A$-band, in the present work we excite the $\mathrm{CH}_{3} \mathrm{I}$ molecule with a short $267 \mathrm{~nm}$ pulse and use a nonresonant $802 \mathrm{~nm}$ probe at a controlled delay to multiphoton ionize the fragments. This nonresonant pulse can, in principle, ionize all species involved in the process, be it parent molecules $\left(\mathrm{CH}_{3} \mathrm{I}\right)$, transient species, and products without any selection on the degree of electronic or vibrational excitation. Figure 1 shows a schematic energy diagram with the excited states discussed above and adiabatic product correlations where the different probing processes are depicted by multiple arrows at different positions of the reaction coordinate. By using velocity map imaging for the ion detection, we can resolve the fragment's kinetic energy release as the reaction evolves into products as well as the spatial anisotropy of the dissociation process. As it will be shown in Secs. III and IV, the present results demonstrate the possibility of employing this technique, where a totally off-resonant probe laser pulse is used, to look at the early-time dynamics in a bond breaking process. We recently published an initial release of the $A$-band photodissociation of $\mathrm{CH}_{3} \mathrm{I}$ using this approach. ${ }^{13}$ 
Here, we present a full account where we have performed further experiments to clarify the role of some effects (molecular alignment, role of pump/probe lasers, etc.) and have extended the study to the deuterated $\mathrm{CD}_{3} \mathrm{I}$ species. We will discuss in length the origin of the findings and the prospects that this technique opens up.

The paper is organized as follows. Section II presents the most relevant experimental aspects relevant to the present work. In Sec. III the results are shown and they are discussed in Sec. IV. Section V closes with the conclusions.

\section{EXPERIMENTAL}

The experimental setup has been described previously ${ }^{8}$ and only the details relevant to the present work will be given here. Briefly, a pump-probe femtosecond laser scheme is used where a Ti:sapphire chirped pulse amplification (CPA) laser system (Spectra-Physics) generates a beam centered at $802 \mathrm{~nm}[80 \mathrm{fs}, 1 \mathrm{~mJ}$ per pulse, $1 \mathrm{kHz}, 16 \mathrm{~nm}$ full width at half maximum (FWHM)], which is then split into two arms in a 80:20 beam splitter. The weaker $0.2 \mathrm{~mJ}$ beam is frequency tripled, obtaining radiation centered at $267 \mathrm{~nm}$ (pump) with a bandwidth of $\sim 2 \mathrm{~nm}$ FWHM. The remaining $0.8 \mathrm{~mJ} 802 \mathrm{~nm}$ beam (probe) is time delayed in a computercontrolled motorized delay stage and later recombined with the pump beam. Typical pulse energies per pulse were 0.1 and $30 \mu \mathrm{J}$ for the pump and probe pulses, respectively, which are controlled with neutral density filters. The polarizations of the laser beams are independently controlled by half wave plates and are used with both polarization axes parallel to the detector, unless otherwise noted, to guarantee cylindrical symmetry in the experiment. Independent telescopes are used to control the focusing geometry of each laser beam on target. The two copropagating beams are focused with a fused silica lens of $f=25 \mathrm{~cm}$ onto a supersonic molecular beam of $\mathrm{CH}_{3} \mathrm{I}$ (or $\mathrm{CD}_{3} \mathrm{I}$ ). Maximum intensities of the pump and probe lasers in the interaction region are estimated to be $\sim 10^{11}$ and $\sim 3 \times 10^{12} \mathrm{~W} \mathrm{~cm}^{-2}$, respectively. For those experiments requiring tunable UV radiation in the range of $304-335 \mathrm{~nm}$, a portion of the fundamental beam is sent to an optical parametric amplifier, where signal and idler pulses are generated in a BBO crystal with wavelengths around 1.2 and $2 \mu \mathrm{m}$, respectively. The signal pulse is later frequency quadrupled to generate the UV pulse.

The vacuum chamber is divided into three sections: source, ionization, and detection. The molecular beam is generated in the source chamber, which is differentially pumped from the other two, by bubbling $\mathrm{He}(1.5$ bar) through a $0{ }^{\circ} \mathrm{C}$ sample of liquid $\mathrm{CH}_{3} \mathrm{I}\left(\mathrm{CD}_{3} \mathrm{I}\right)$. The gas sample is expanded into the vacuum chamber via a homemade $1 \mathrm{kHz}$ piezoelectric valve ${ }^{14}$ and the gas pulse is skimmed into the ionization vacuum chamber. In order to avoid clusters in the molecular beam, the interaction between the laser pulses and the molecular beam is fixed at the rising edge of the gas pulse. The skimmed molecular beam is entrained into the region between the repeller and extractor plates of an ion lens system located in the ionization chamber perpendicular to the propagation of the molecular beam and working in velocity map configuration. ${ }^{15}$ Ions produced in the interaction region are extracted into the $50 \mathrm{~cm}$ timeof-flight tube and detected by a dual microchannel plate (MCP) in Chevron configuration coupled to a phosphor screen. The MCP output signal could be visualized on a 600 $\mathrm{MHz}$ digital oscilloscope. For ion imaging detection, mass selection is performed at the detector by fast gating the gain of the first MCP plate. The two-dimensional (2D) images on the phosphor screen are recorded by a 12-bit charge coupled device camera. Typical acquisition times for each image are $30 \mathrm{~s}$, equivalent to accumulating over $3 \times 10^{4}$ laser shots. Images were acquired automatically for series of pumpprobe delay times. Raw images were Abel inverted using the pBasex method. ${ }^{16}$

The detector is calibrated by an "on-resonant" pumpprobe experiment of $\mathrm{CH}_{3} \mathrm{I}$ photodissociation using a $267 \mathrm{~nm}$ pump laser and a $333.5 \mathrm{~nm}$ probe of free $\mathrm{CH}_{3}$ fragments by $2+1$ REMPI at long time delay as described elsewhere. ${ }^{7,8}$

\section{RESULTS}

\section{A. Transients measured by conventional time-of-flight mass spectrometry}

When using conventional time-of-flight mass spectrometry (TOFMS) in combination with the femtosecond laser pump-probe technique, the different ion mass peaks measured in the trace of the digital oscilloscope are integrated as a function of the delay time between the pump and probe laser pulses. A careful kinetics analysis of the measured transients is then used to extract dynamical information about the process under study. In the present work, we have measured TOF mass spectra of the ions resulting from femtosecond laser irradiation of $\mathrm{CH}_{3} \mathrm{I}$ at 267 and $802 \mathrm{~nm}$. These mass spectra contain three different ion peaks, which correspond to the fragments $\mathrm{CH}_{3}{ }^{+}$and $\mathrm{I}^{+}$, and to the parent $\mathrm{CH}_{3} \mathrm{I}^{+}$. By integration of each mass peak as the pump-probe time delay is changed, the temporal evolution of each ion signal can be monitored.

The results obtained for $\mathrm{CH}_{3} \mathrm{I}$ and its isotopologue $\mathrm{CD}_{3} \mathrm{I}$ are shown in Fig. 2 after subtraction of the single pump (267 $\mathrm{nm})$ and probe $(802 \mathrm{~nm}$ ) laser signals (we have worked in conditions where the maximum pump-probe signal is $\sim 6$ times larger than the signals produced by the two laser beams separately). Positive delay times in the plots mean that the probe laser $(802 \mathrm{~nm})$ arrives after the pump (267 nm), whereas the inverse is true for negative delay times. When the pump-probe delay time is small, a strongly enhanced ion signal is observed for all ions; this enhanced signal disappears after a positive delay time of about $300 \mathrm{fs}$, although a much weaker pump-probe signal remains for at least $100 \mathrm{ps}$ for $\mathrm{CH}_{3}{ }^{+}\left(\mathrm{CD}_{3}{ }^{+}\right)$and $\mathrm{I}^{+}$. This weaker component will be more clearly shown and discussed later. In the case of the parent ion, there is no detectable pump-probe signal away from the enhancement at short delay times. Figure 2 also shows that although the shape of the transients is similar for the different species (Gaussian-like), their temporal behavior is not identical. The main difference between the different transients is a positive temporal shift observed for the fragment ions with respect to the parent ion. This shift is around 70 fs for $\mathrm{CH}_{3}{ }^{+}\left(\mathrm{CD}_{3}{ }^{+}\right.$) and 40 fs for $\mathrm{I}^{+}$(with negligible 

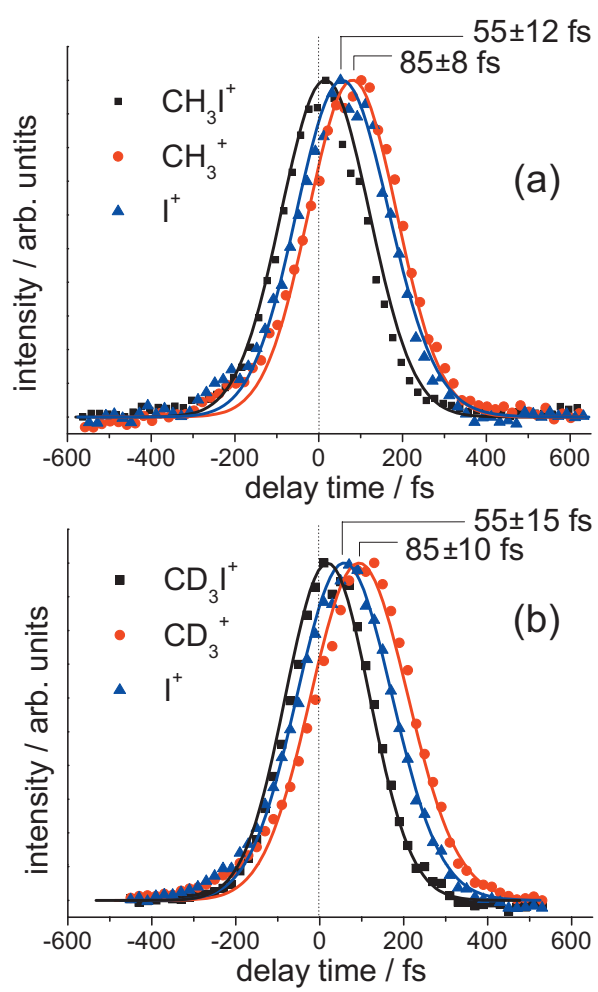

FIG. 2. Pump-probe transient ion signal of the parent (a) $\mathrm{CH}_{3} \mathrm{I}^{+}$and (b) $\mathrm{CD}_{3} \mathrm{I}^{+}$and their corresponding fragments $\mathrm{CH}_{3}{ }^{+}, \mathrm{CD}_{3}{ }^{+}$, and $\mathrm{I}^{+}$obtained by integrating each mass peak in the oscilloscope trace. The different transients in each plot are normalized for clarity of presentation. The lines correspond to fits to an exponential decay convoluted with a Gaussian representing the cross correlation (FWHM of $210 \pm 30 \mathrm{fs}$ ) between the pump $(267 \mathrm{~nm})$ and probe $(802 \mathrm{~nm})$ pulses. For $\mathrm{CH}_{3} \mathrm{I}$, the decay times found for parent, $\mathrm{CH}_{3}{ }^{+}$, and $\mathrm{I}^{+}$are $20 \pm 10,50 \pm 20$, and $40 \pm 20 \mathrm{fs}$, respectively. For $\mathrm{CD}_{3} \mathrm{I}$, the decay times for parent, $\mathrm{CD}_{3}{ }^{+}$, and $\mathrm{I}^{+}$are $15 \pm 10,50 \pm 20$, and $30 \pm 20 \mathrm{fs}$, respectively. Time shifts between the parent ion and the fragment ion transients are found to be $40 \pm 10$ and $70 \pm 8$ fs for $\mathrm{CH}_{3}{ }^{+}$and $\mathrm{I}^{+}$, respectively, and $40 \pm 15$ and $70 \pm 10 \mathrm{fs}$ for $\mathrm{CD}_{3}{ }^{+}$and $\mathrm{I}^{+}$, respectively. Time zero is defined as the pump-probe delay time corresponding to the instantaneous rise of the exponential decay function used to fit the corresponding parent ion transient. It must be emphasized that given the width of the cross correlation, asymmetric transients would only be obtained for decay times $>50$ fs.

isotope effect). All the transients shown in Fig. 2 have been fitted using an exponential decay molecular response function starting at a given delay time (to account for the possible contribution of intermediate states with a measurable lifetime), convoluted by a Gaussian function of parametrized width (representing the cross correlation profile of the 267 and $802 \mathrm{~nm}$ laser pulses). We have defined "zero" delay time as the pump-probe delay time corresponding to the instantaneous rise of the exponential decay function used to fit the parent ion transient. The weak long delay time component of the signal for the fragment ions has been ignored for this first analysis. As can be inferred from visual inspection, no asymmetries are observable in the transients, and a Gaussian function correctly fits all experimental curves. Gaussian widths of $210 \pm 30$ fs provide the best fit, and we can only provide an upper limit of $50 \pm 20 \mathrm{fs}$ for the time constant of the exponential decays of the $\mathrm{CH}_{3}\left(\mathrm{CD}_{3}\right)$ fragments and of $40 \pm 20 \mathrm{fs}$ for the I fragment.

Janssen et al. ${ }^{17}$ observed the decay of the $\mathrm{CH}_{3} \mathrm{I}$ and $\mathrm{CD}_{3} \mathrm{I}$ $6 p^{2} E_{1 / 2}$ and $7 s^{2} E_{3 / 2}$ Rydberg states accessed by two-photon femtosecond excitation at wavelengths in the range of 300$316 \mathrm{~nm}$ by the measurement of parent ion transients using a nonresonant probe pulse at $618 \mathrm{~nm}$ and TOFMS. They proposed that in the competition established between excitation to the $A$-band and further absorption into high-lying Rydberg states, their conditions strongly favored multiphoton absorption due to the ultrashort character of the excitation laser pulses and the relatively high intensities used. They assigned the measured lifetimes to predissociation rates of the accessed Rydberg states. The pump laser used in our work (267 $\mathrm{nm}$ ) with a two-photon energy of $9.275 \mathrm{eV}$ and a bandwidth of around $0.05 \mathrm{eV}$ is at close resonance with several higherlying members of Rydberg series of $\mathrm{CH}_{3} \mathrm{I}$ (Ref. 18) with $10 p$, $9 d$, and $10 d$ (of the Rydberg series converging to the first ionization potential at $9.54 \mathrm{eV},{ }^{2} E_{3 / 2}$ ground state of the ion) and $8 s$ and $6 d$ (of the Rydberg series converging to the second ionization potential at $10.17 \mathrm{eV},{ }^{2} E_{1 / 2}$ state of the ion) being the possible contributors. In analogy with Janssen et al. ${ }^{17}$ a possible mechanism for the parent ion transient observed at short delay time is a resonant two-photon transition to these higher-lying Rydberg states, followed by ionization with one or more $802 \mathrm{~nm}$ photons. In this case, the decay of the parent ion signal would be a measurement of the dissociation rate of the corresponding Rydberg states. Decay times for several Rydberg states ( $6 p$ and $7 s)$ were measured in Ref. 17 with values ranging from 200 to $100 \mathrm{fs}$ in the pump wavelength range between 316 and $300 \mathrm{~nm}$, respectively, and with a further decrease in decay times as shorter wavelengths were used. In principle, this would be compatible with our observations, since for the higher-lying Rydberg states accessed by two-photon $267 \mathrm{~nm}$ excitation, the lifetimes could be well below our 50 fs detection limit. However, a strong isotope effect was observed in Ref. 17, with the lifetimes of the $\mathrm{CD}_{3} \mathrm{I}$ Rydberg states increasing in some cases by a factor of about 4 with respect to those of $\mathrm{CH}_{3} \mathrm{I}$. The decay times in our case are not only below the present temporal resolution $(<50 \mathrm{fs})$ for $\mathrm{CH}_{3} \mathrm{I}$, but apparently also for $\mathrm{CD}_{3} \mathrm{I}$.

As shown in Fig. 2, the shape of the $\mathrm{CH}_{3}\left(\mathrm{CD}_{3}\right)$ and I fragment ion transients is quite similar to those measured for the parent ion, $\mathrm{CH}_{3} \mathrm{I}$ or $\mathrm{CD}_{3} \mathrm{I}$. The most important feature of the fragment ion transients in comparison with those of the parent ion is the time shift to positive delay times of 70 and 40 fs for the $\mathrm{CH}_{3}\left(\mathrm{CD}_{3}\right)$ and I fragments, respectively. The fast transients of the fragment ions can originate from the dissociation of the parent ion (dissociative ionization) or by MPI of neutral fragments after excited state photodissociation of the parent molecule. It is hard to infer from the measured transients obtained just by integrating all velocity components of the fragment peaks in the mass spectra what is(are) the process(es) responsible for them if in addition we have a simple kinetics represented by a fast decay and a time shift. Thus, it is mandatory to resolve the kinetic energy and spatial anisotropy of the fragments to obtain additional information.

\section{B. Velocity map imaging of the $\mathrm{CH}_{3}$ fragment}

In order to elucidate the mechanisms contributing to the enhanced signal measured at short delay times between the 


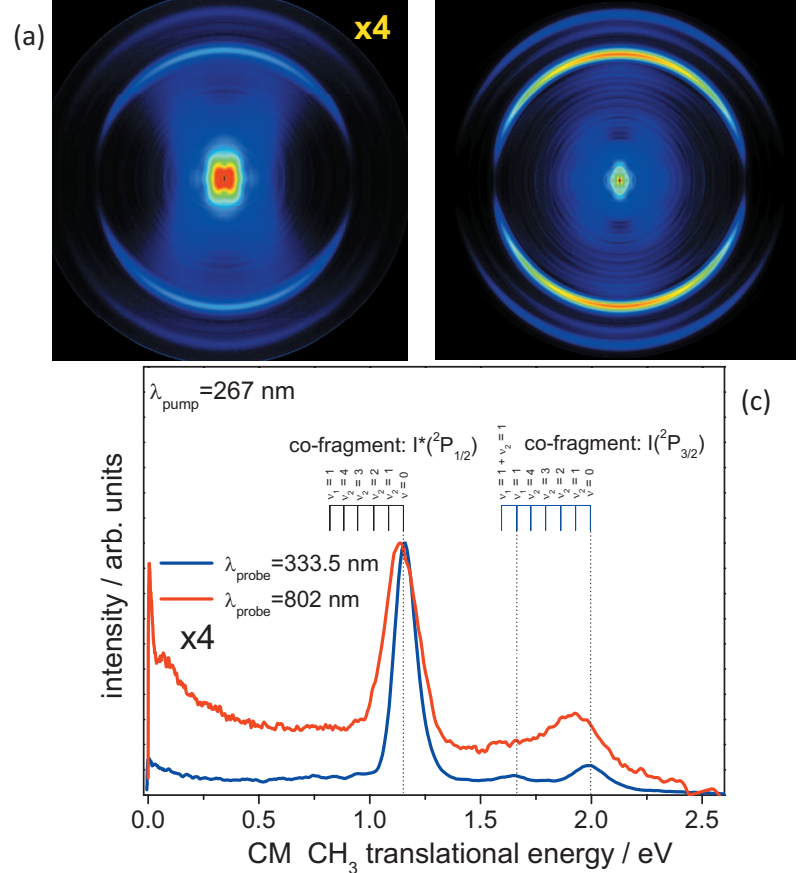

FIG. 3. Abel-inverted $\mathrm{CH}_{3}{ }^{+}$images obtained upon excitation of $\mathrm{CH}_{3} \mathrm{I}$ at 267 $\mathrm{nm}$ for a pump-probe delay time of $1 \mathrm{ps}$. (a) $\mathrm{CH}_{3}$ detection by nonresonant MPI at $802 \mathrm{~nm}$ (at least seven photons are needed). The contrast of this image has been increased by a factor of 4 . (b) $\mathrm{CH}_{3}$ detection by $2+1$ REMPI at $333.5 \mathrm{~nm}\left[Q\right.$ branch of the $3 p_{z}\left({ }^{2} A_{2}^{\prime \prime} \leftarrow{ }^{2} A_{2}^{\prime \prime}\right) 0^{0}{ }_{0}$ transition]. (c) Asymptotic $\mathrm{CM} \mathrm{CH}_{3} \mathrm{KEDs}$ obtained at 802 (red) and $333.5 \mathrm{~nm}$ (blue) probe wavelengths together with the assignments made for the different product channels. The curves have been normalized to clarify the comparison.

pump and probe pulses, we have used the velocity map imaging technique for the $\mathrm{CH}_{3}$ fragments as a function of delay time to obtain complete information on the energy release and spatial anisotropy of the possible processes by which fragment ions are produced. Figure 3(a) shows an Abelinverted $\mathrm{CH}_{3}{ }^{+}$image taken in a situation where the pump pulse precedes the probe by $1 \mathrm{ps}$. Identical images appear at longer delay times, so that we will refer to this as the "asymptotic" case. Apart from a component in the center of the image that will be discussed later, this image shows two distinct rings with characteristic widths and anisotropies. They are directly attributable to the two channels open to $\mathrm{CH}_{3} \mathrm{I}$ in the $A$-band photodissociation at $267 \mathrm{~nm}$, leading to $\mathrm{CH}_{3}$ plus either ground state $\mathrm{I}\left({ }^{2} P_{3 / 2}\right)$ or spin-orbit excited $\mathrm{I}^{*}\left({ }^{2} P_{1 / 2}\right)$, which will be referred to as the I or I* channels, respectively. For comparison, Fig. 3(b) shows an image where the femtosecond probe laser is tuned at $333.5 \mathrm{~nm}$, which corresponds to the $Q$ branch of the $3 p_{z}\left({ }^{2} A_{2}^{\prime \prime}\right.$ $\left.\leftarrow^{2} A_{2}^{\prime \prime}\right) 0_{0}^{0}$ transition for a two-photon process followed by absorption of a third photon to resonantly ionize $\mathrm{CH}_{3}(\nu=0){ }^{7,8}$ Despite the similarities, an important difference must be noted between the two images. Whereas in the latter case, the resonant probe pulse selects a particular vibrational state of $\mathrm{CH}_{3}$ (the vibrational ground state), for which $2+1$ REMPI is produced, in the former case the probe laser centered at $802 \mathrm{~nm}$ is nonresonant, and therefore induces nonselective MPI of all $\mathrm{CH}_{3}$ fragments regardless of their degree of vibrational excitation. It is important to notice that the signal obtained in the resonant experiment is much higher than that obtained in the nonresonant experiment since REMPI is a much more efficient process than MPI. The important fact, however, is that velocity map imaging is a very sensitive technique allowing MPI detection of neutral fragments arising from the photodissociation on a repulsive electronic excited state of a neutral molecule.

Figure 3(c) shows a comparison between the kinetic energy distribution (KED) extracted from angular integration of the asymptotic image in the nonresonant MPI probe case and that resulting from angular integration of the experiment with REMPI detection of $\mathrm{CH}_{3}$. In the second case, the probe laser resonantly ionizes vibrationless $\mathrm{CH}_{3}$, and it is clear from the figure that only $\nu=0$ states are detected (except an accidental detection of $\nu_{1}=1$ due to the overlap of the $1^{1}{ }_{1}$ transition with the bandwidth of the probe laser). This is in contrast with the nonresonant MPI probe at $802 \mathrm{~nm}$, where several $\nu_{2}$ states are observed (the peaks are broader), especially in correlation with the I channel, where population inversion occurs (as had been previously reported, for instance, in Ref. 19). It is interesting to note that under the assumption that all vibrational states of methyl have the same ionization probability by the nonresonant probe laser, this result provides a direct measurement of the nascent vibrational populations.

Abel-inverted $\mathrm{CH}_{3}{ }^{+}$images as a function of delay time are shown in Fig. 4 The first image, corresponding to the probe pulse preceding the pump by $450 \mathrm{fs}$, shows a signal that corresponds to $\mathrm{CH}_{3}{ }^{+}$resulting from the interaction with each of the pulses separately; such image contains no contribution of the pump-probe signal and is more concentrated around the center of the image, i.e., low kinetic energy. This type of contribution typically derives from dissociative ionization processes where the electron carries most of the available energy and fragment ions appear with low kinetic energies. All the other images taken at later delay times contain this "background" or low kinetic energy contribution.

The most important result shown in Fig. 4 is that for delay times close to zero, the images show enhanced rings with a global structure in strong similarity with that corresponding to the asymptotic case, containing strong contribution of ions in the same energy regions as the asymptotic I and I* channels, with non totally dissimilar ratio and anisotropy. Due to this similarity, we will from now on name these two rings as the I and $\mathrm{I}^{*}$ channels, even though we will discuss their origin later. The distribution of ions near temporal overlap also shows clear differences with respect to the asymptotic case as follows: (i) an enhanced intensity near the temporal overlap between the pump and probe pulses; (ii) a different ratio between the I and I* channels and changes in their KEDs; (iii) a significant appearance of a new strongly anisotropic ring, which corresponds to a lower energy channel; and (iv) an overall increased anisotropy in the I and I* channels.

We will first analyze the temporal behavior of each of the detected components of the $\mathrm{CH}_{3}{ }^{+}$images. As it has been shown in the images of Fig. 4, we can consider three main contributions: two rings appearing in the energy regions of $\sim 1.15$ and $\sim 2.00 \mathrm{eV}$, similar to the asymptotic rings, and a new lower energy ring appearing at $\sim 0.6 \mathrm{eV}$. We have in- 


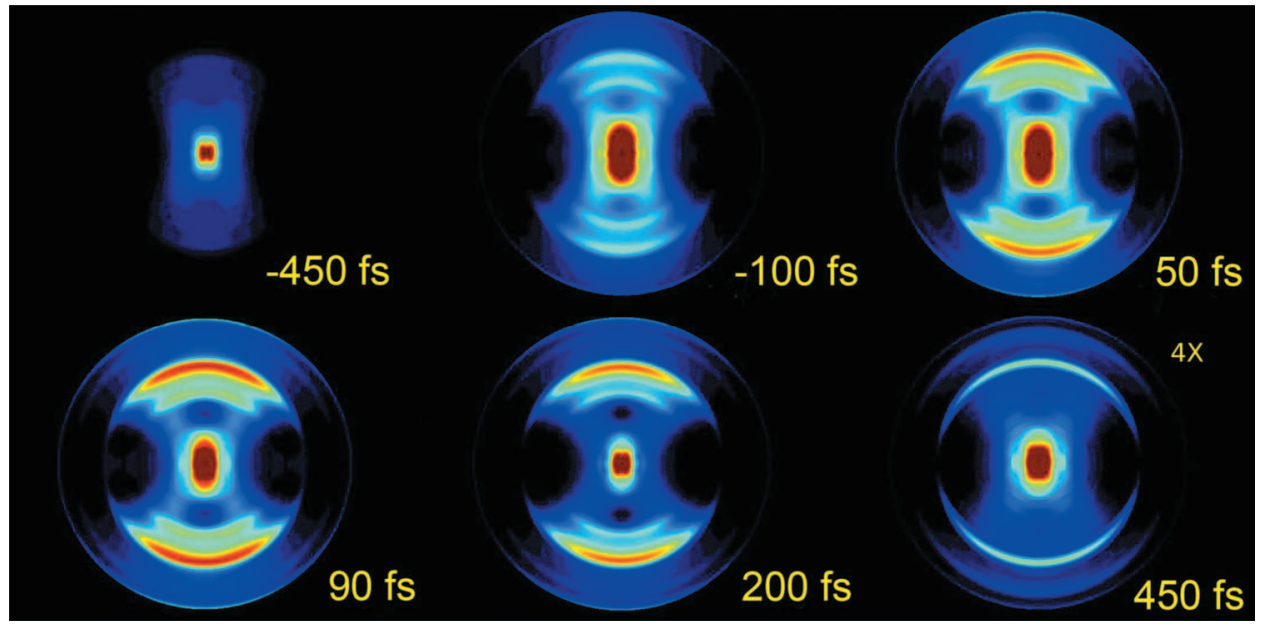

FIG. 4. False-color Abel-inverted $\mathrm{CH}_{3}{ }^{+}$images obtained as a function of pump-probe delay time. Color scale is kept constant so as to highlight the changes in overall signal intensity. The image corresponding to the asymptotic case (delay time of $+450 \mathrm{fs}$ ) is intensified by a factor of 4 with respect to the others. The most intense ring corresponds to the main $\mathrm{CH}_{3}+\mathrm{I}^{*}\left({ }^{2} P_{1 / 2}\right)$ dissociation channel. A larger radius ring, which produces a much weaker signal, can be attributed to the $\mathrm{CH}_{3}+\mathrm{I}\left({ }^{2} P_{3 / 2}\right)$ channel. An additional channel of lower kinetic energy (smaller radius) and strong anisotropy can be seen when pump and probe pulses overlap. The weak but clear rings that can be observed in the image taken at a delay of +450 fs corresponding to the asymptotic $\mathrm{CH}_{3}$ $+\mathrm{I}^{*}\left({ }^{2} P_{1 / 2}\right)$ and $\mathrm{CH}_{3}+\mathrm{I}\left({ }^{2} P_{3 / 2}\right) A$-band dissociation channels keep a similar intensity for much longer delay times $(>450 \mathrm{fs})$.

tegrated each energy zone separately as a function of delay time, and the results are shown in Figs. 5(b)-5(d), respectively. Determination of time zero has been possible by simultaneously measuring a transient for the parent $\mathrm{CH}_{3} \mathrm{I}^{+}$signal. This was performed by reducing the detection gating discrimination, causing a situation where the intense peak corresponding to the parent ion was partially detected overlapped on the $\mathrm{CH}_{3}{ }^{+}$image. This did not produce confusion, since the spatial localization of the parent ion in the image was in a zone where no contribution of the methyl ion fragment is present.

As can be seen in Fig. 5, the I and $\mathrm{I}^{*}$ channels show a very similar temporal profile with a delay on the rising edge of about 85 fs and a fast decrease that ends up in a plateau. This plateau corresponds to the asymptotic process already mentioned, i.e., a highly multiphotonic ionization probe process of the free $\mathrm{CH}_{3}$ fragment induced by the intense $802 \mathrm{~nm}$ probe pulse. The curves shown in Figs. 5(b) and 5(c) have been fitted with the convolution of a Gaussian (simulating the instrumental response function due to the finite duration of the laser pulses) with a molecular function that is the result of adding an exponential decay to a Boltzmann sigmoidal curve of the form $S \propto\left\{1+\exp \left[\left(t-t_{0}\right) / t_{C}\right]\right\}^{-1}$ (Ref. 8) (where $t_{0}$ is the center temporal position and $t_{C}$ describes the steepness of the rise). The latter simulates the asymptotic behavior. In the case of the curves shown in Figs. 5(a) and 5 (d) the molecular function is just an exponential decay. As was the case with the data presented in Fig. 2 only an upper limit for the exponential time constant could be obtained $(<50 \mathrm{fs})$ given the width of the cross correlation function.

The peak observed at $0.6 \mathrm{eV}$ corresponding to the inner anisotropic ring shows a different time profile, somewhat narrowed with respect to the parent ion transient and with no delay or plateau. This suggests that this channel is related to a highly nonlinear process, possibly with respect to both the pump and the probe pulses. This is in agreement with power dependence measurements that will be described below.

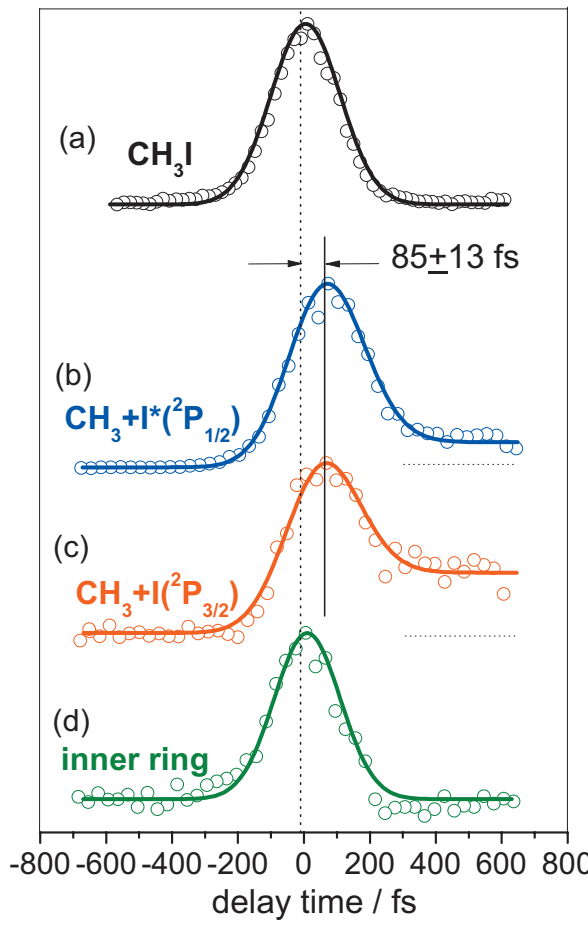

FIG. 5. Transients obtained by angular integration of the different rings observed in the $\mathrm{CH}_{3}^{+}$images. (a) $\mathrm{CH}_{3} \mathrm{I}^{+}$transient. The $\mathrm{CH}_{3} \mathrm{I}^{+}$signal was extracted from the images as indicated in the text. (b) Ring assigned to the $\mathrm{CH}_{3}+\mathrm{I}^{*}\left({ }^{2} P_{1 / 2}\right)$ channel. (c) Ring assigned to the $\mathrm{CH}_{3}+\mathrm{I}\left({ }^{2} P_{3 / 2}\right)$ channel. (d) Inner ring. The $\mathrm{CH}_{3} \mathrm{I}^{+}$transient sets the time zero in the pump-probe experiment. Note that the transients shown in (b) and (c) have the same delay time at maximum intensity of $85+13$ fs with respect to time zero. The transient shown in (d) has no delay time at maximum. Open circles: experimental data. Solid lines: fits to a molecular response function convoluted with a Gaussian representing the cross correlation (FWHM of $220 \pm 30$ fs) between the pump $(267 \mathrm{~nm})$ and probe $(802 \mathrm{~nm})$ pulses. For the $\mathrm{CH}_{3} \mathrm{I}^{+}$and inner ring transients, the molecular function is an exponential decay with a decay time of $20 \pm 10$ fs. For (b) and (c) transients the molecular function is made of an exponential decay added to a Boltzmann sigmoidal. The parameters of the fits are $30 \pm 20 \mathrm{fs}$ for the decay time and $t_{0}=110 \mathrm{fs}$ and $t_{C}$ $=20 \pm 10$ fs for the sigmoidal curve. The ratios between the amplitudes of the exponential decay to the sigmoidal are 49 and 19 for transients (b) and (c), respectively. 

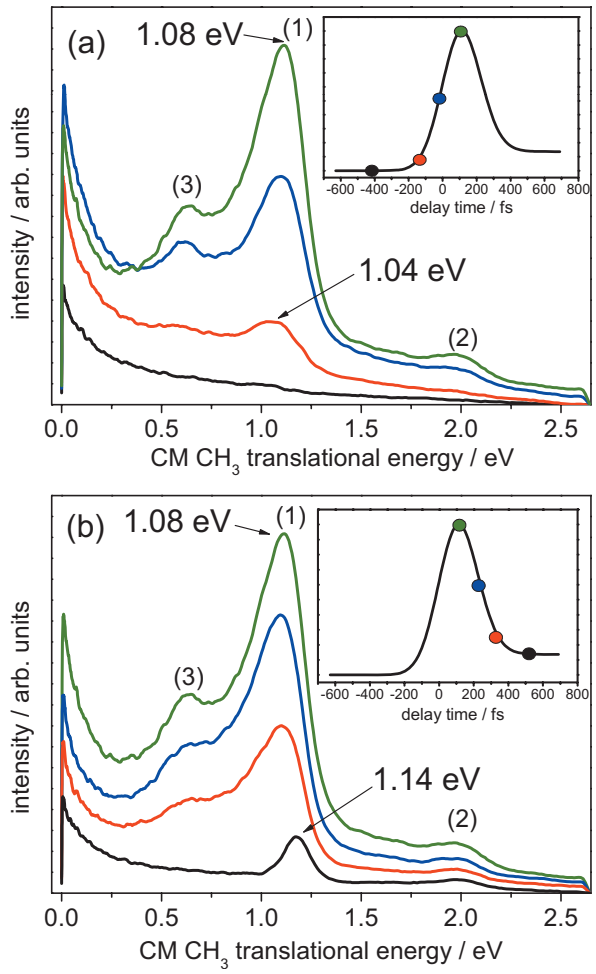

FIG. 6. $\mathrm{CM} \mathrm{CH}_{3} \mathrm{KEDs}$ at selected pump-probe delay times as indicated in the insets, where the fitted curve corresponding to the transient shown in Fig. 5(b) is depicted along with color circles to clarify what delay times are represented: (a) from -400 to 110 fs and (b) from 110 to 520 fs. Peaks (1) and (2) correspond to the $\mathrm{CH}_{3}+\mathrm{I}^{*}\left({ }^{2} P_{1 / 2}\right)$ and $\mathrm{CH}_{3}+\mathrm{I}\left({ }^{2} P_{3 / 2}\right)$ channels, respectively. Peak (3) may correspond to a dissociative ionization channel. The labels on top of peak (1) indicate the values of the $\mathrm{CH}_{3}$ kinetic energy at the maximum of the peak.

The behavior of the KEDs obtained from the $\mathrm{CH}_{3}{ }^{+}$images will now be presented as a function of the pump-probe delay time. Figure 6 shows the center-of-mass $(\mathrm{CM}) \mathrm{CH}_{3}$ KEDs resulting from the angular integration of the Abelinverted images at different delay times. The KED for a delay time of about -400 fs [black in Fig. 6(a)] simply shows the background ion signal corresponding to the addition of the contributions of the two laser pulses separately. The asymptotic curve [black in Fig. 6(b), delay time of about +500 fs, already shown in Fig. 3(c)] shows two peaks [labeled (1) and (2)], corresponding to the $\mathrm{CH}_{3}+\mathrm{I}^{*}\left({ }^{2} P_{1 / 2}\right)$ and $\mathrm{CH}_{3}+\mathrm{I}\left({ }^{2} P_{3 / 2}\right) A$-band photodissociation channels. The intermediate distributions taken close to the temporal overlap between the pump and probe pulses show the presence of what appear to be the same two asymptotic channels, except somewhat shifted toward lower energies and broadened in energy. The shift toward lower energy values diminishes as the delay time is increased, until it disappears at around $+400 \mathrm{fs}$, where the asymptotic situation is fully recovered. From that delay time onward, the $\mathrm{CH}_{3}+\mathrm{I}^{*}\left({ }^{2} P_{1 / 2}\right)$ and $\mathrm{CH}_{3}$ $+\mathrm{I}\left({ }^{2} P_{3 / 2}\right) A$-band photodissociation channels can be distinguished at 1.14 and $1.92 \mathrm{eV}$, respectively. Figure 7 depicts the $\mathrm{CM} \mathrm{CH}_{3}$ kinetic energy corresponding to the maximum of the distribution for the $\mathrm{I}^{*}$ channel [the peak around 1.10 $\mathrm{eV}$, labeled (1) in Fig. 6] as a function of delay time. As can be seen, the kinetic energy of the maximum of the main peak in the KED decreases as we move back in time from the

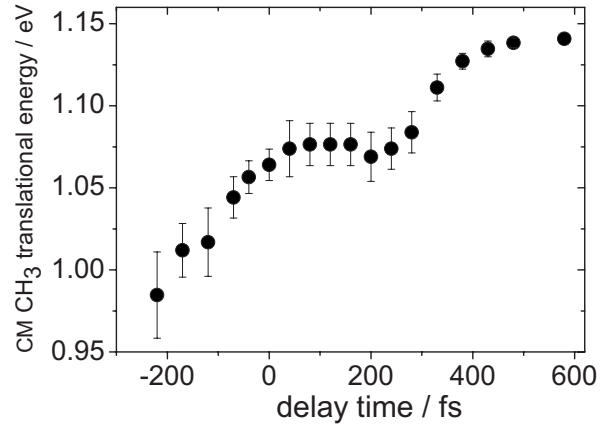

FIG. 7. Representation of the $\mathrm{CM} \mathrm{CH}_{3}$ kinetic energy at the maximum of peak (1) as a function of the pump-probe delay time. The error bars on each point represent the standard deviation of a number of sequences of images.

asymptotic value of $1.14 \mathrm{eV}$ toward negative delays (with a shallow minimum and a leveloff around zero) to continue decreasing at negative delay times to values less than $1.0 \mathrm{eV}$. A similar behavior is found for the I channel [the small signal around $2 \mathrm{eV}$, labeled (2) in Fig. 6], although it is less clear due to the poorer signal-to-noise ratio. Finally, the additional channel not attributable to the $A$-band photodissociation, appearing at lower kinetic energies of $0.6 \mathrm{eV}$, is clearly shorter lived than the others, and of a multiphoton nature on both the pump and probe laser pulses. The possible assignment of this peak will be discussed later.

The Abel-inverted images also contain information on the angular distribution of fragments for each energy channel. This can be done through integration of the images across the radii corresponding to each of the rings, which can be plotted as a function of polar angle. For a one-photon dissociation process, we expect an angular dependence of the form

$$
\mathrm{I}(\theta)=\frac{\sigma}{4 \pi}\left[1+\beta_{2} P_{2}(\cos \theta)\right],
$$

where $\sigma$ is the total absorption cross section, $\theta$ is the angle between the polarization axis of the photolysis laser and the fragment velocity vector, $\beta_{2}$ is the anisotropy parameter, and $P_{2}(\cos \theta)$ is the second Legendre polynomial. For multiphoton transitions, it is necessary to expand the series to include Legendre polynomials of higher order,

$$
\mathrm{I}(\theta)=\frac{\sigma}{4 \pi}\left[1+\beta_{2} P_{2}(\cos \theta)+\beta_{4} P_{4}(\cos \theta)+\cdots\right],
$$

where $P_{4}(\cos \theta)$ is the Legendre polynomial of fourth order and $\beta_{4}$ is the second-order anisotropy parameter.

Figure 8 shows the values of $\beta_{2}$ and $\beta_{4}$ obtained from the Abel-inverted images for channels I* and I as a function of delay time (the fitted transient profiles for each channel of Fig. 5 are included for clarity as reference for better viewing). At the asymptotic delay times, all angular distributions can be correctly fitted with Eq. (3) with a $\beta_{2}$ of $1.9 \pm 0.1$ and $1.6 \pm 0.1$ for the I* and I channels, respectively, matching the values of previous works at $267 \mathrm{~nm} .{ }^{8,20}$ Contrarily, for the region around temporal overlap it is not possible to fit the angular distributions with Eq. (3), and thus Eq. (4) is used instead. It is clear that a more pronounced anisotropy is seen in the enhancement signals than in the asymptotic ones, as 

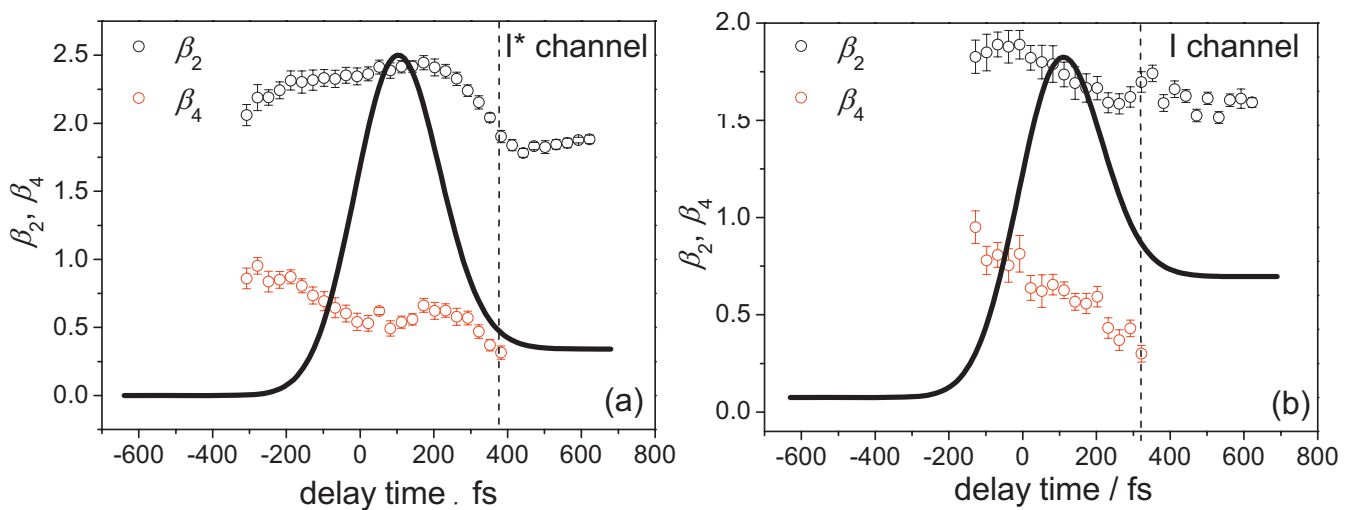

FIG. 8. $\beta_{2}$ (black dots) and $\beta_{4}$ (red dots) anisotropy parameters as a function of the pump-probe delay time obtained by radial integration of the images for the (a) $\mathrm{CH}_{3}+\mathrm{I}^{*}\left({ }^{2} P_{1 / 2}\right)$ and (b) $\mathrm{CH}_{3}+\mathrm{I}\left({ }^{2} P_{3 / 2}\right)$ channels. Error bars on each point correspond to the standard deviation of a number of sequences of images. For clarity the fitted transients shown in Fig. 5(b) and 5(c) are depicted in each panel. Vertical dashed lines indicate the limit from where the angular distributions can be fitted by using only $\beta_{2}$ (asymptotic case). See the text for more details.

shown by the increase in the value of $\beta_{2}$ to $2.3 \pm 0.3$ and $1.8 \pm 0.2$ for the I* and I channels, respectively, and the positive values of $\beta_{4}$.

The stronger anisotropy at short delay times can be characterized by the average of the $\cos ^{2} \theta_{2 \mathrm{D}}$ weighted by the population for each (2D) laboratory angle $\theta_{2 \mathrm{D}}$ (i.e., the polar angle measured from the direction of the polarization axis on the plane of the imaging detector) for a given energy region, i.e., $\left\langle\cos ^{2} \theta_{2 \mathrm{D}}\right\rangle,{ }^{21}$ for each channel as a function of the delay time. The $\left\langle\cos ^{2} \theta_{2 \mathrm{D}}\right\rangle$ angular distributions corresponding to the different channels are considerably narrower near the temporal overlap of the pump-probe lasers (not shown). Two effects could be contributing to this narrowing of the angular distribution at time zero. One would imply that the absorption is no longer a one-photon process for the pump pulse. Multiphoton processes can have narrower angular distributions, according to Eq. (4). The other effect would be related to a dynamic alignment process of the parent molecule due to the intense $802 \mathrm{~nm}$ pulse (see for instance Ref. 22), which would concentrate the probability of finding the molecular axis near the vertical axis (vertical polarization). This would then be reflected on the ion images as a narrowing of the angular distribution around the $\theta_{2 \mathrm{D}}$ angle. ${ }^{21}$ In order to separate these effects, a further three laser pulse experiment was performed where impulsive molecular alignment was induced using an equivalent (same energy per pulse and focusing conditions) $802 \mathrm{~nm}$ pulse. After a controlled delay, a photodissociation experiment was performed at $267 \mathrm{~nm}$, followed by an on-resonance, long delay time REMPI probing of the resulting methyl fragment (as described in Ref. 8). This experiment is similar to that carried out by Stapelfeldt and co-workers ${ }^{21}$ for $\mathrm{CH}_{3} \mathrm{I}$, detecting in that case the $\mathrm{I} / \mathrm{I}^{*}$ fragment by $2+1$ REMPI using a long time delayed nanosecond laser pulse. The results are shown in Fig. 9, where the $\left\langle\cos ^{2} \theta_{2 \mathrm{D}}\right\rangle$ value is plotted as a function of the delay time between the alignment pulse and the dissociation pulse. That we do obtain some degree of molecular alignment is proven by the fact that, at the first half revival of $\mathrm{CH}_{3} \mathrm{I}$ (which happens at around $32 \mathrm{ps}$ ), we do see the characteristic variations in the angular distributions. However, the change in $\left\langle\cos ^{2} \theta_{2 \mathrm{D}}\right\rangle$ is around a factor of 4 lower than that observed at time zero, whereas it is predicted that if molecular alignment was the only source of angular narrowing, the variations at the half revival and at time zero should be of the same order. Therefore, the results indicate that even though molecular alignment does play a partial role in the angular narrowing observed at time zero, the main attribution must be to a change in the nature of the pump laser absorption from a one-photon to a multiphoton process.

We would like to stress here that at time zero there is no distinction between the pump and the probe laser pulses, so that the $802 \mathrm{~nm}$ beam can also be considered as "pump" in this situation. An experiment where the polarization of the $802 \mathrm{~nm}$ beam was rotated by $90^{\circ}$ was carried out to clarify this issue. For the asymptotic case (delay time of $>400 \mathrm{fs}$ ), the polarization of the probe laser does not play a significant role on the KEDs of the $\mathrm{CH}_{3}$ fragments or their anisotropy. This is consistent with the picture where the $802 \mathrm{~nm}$ beam acts as a pure probe of the methyl fragment, exclusively

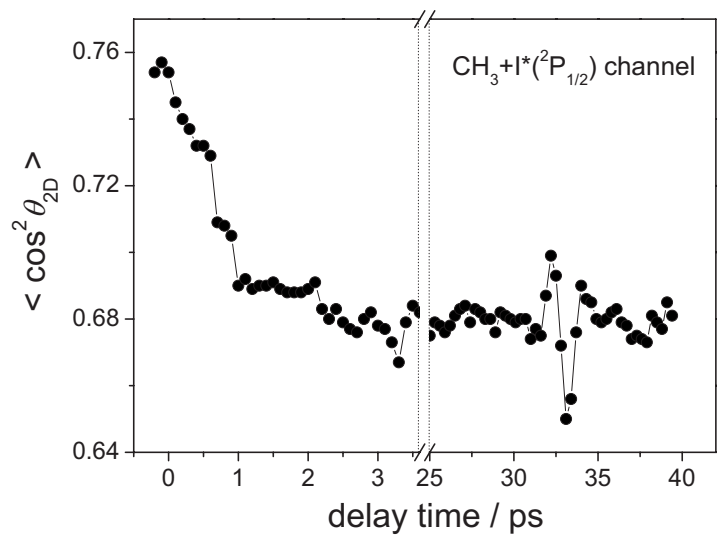

FIG. 9. Alignment dynamics of $\mathrm{CH}_{3} \mathrm{I}$ represented by $\left\langle\cos ^{2} \theta_{2 \mathrm{D}}\right\rangle$. This quantity is derived from the intense ring in the $\mathrm{CH}_{3}{ }^{+}$raw images assigned to the $\mathrm{CH}_{3}(\nu=0)+\mathrm{I}^{*}\left({ }^{2} P_{1 / 2}\right)$ channel measured as a function of the delay time between an IR alignment pulse $(802 \mathrm{~nm})$ and the excitation pulse $(267 \mathrm{~nm})$. The $\mathrm{CH}_{3}(\nu=0)$ fragment arising from the $A$-band photodissociation of $\mathrm{CH}_{3} \mathrm{I}$ is probed at a long delay time (several picoseconds) by $2+1$ REMPI at $333.5 \mathrm{~nm}\left[Q\right.$ branch of the $3 p_{z}\left({ }^{2} A_{2}^{\prime \prime} \leftarrow{ }^{2} A_{2}^{\prime \prime}\right) 0^{0}{ }_{0}$ transition]. The first half revival is observed at a delay time between the alignment and photolysis pulses of about $32 \mathrm{ps}$, which corresponds to half of the rotational period of the molecule. 
producing MPI (the $\mathrm{CH}_{3}{ }^{+}$recoil in this ionization process is negligible since the electron carries practically all the available energy). The situation changes completely for delay times close to temporal overlap. In this case, even though the overall signal does suffer from an enhancement of similar magnitude and with similar temporal behavior, the clear rings that could be detected for parallel polarizations are no longer visible, and instead, an intense unstructured contribution is detected around the center of the image. The essential conclusion that can be derived from these measurements is that the $802 \mathrm{~nm}$ laser does behave as pump laser for the enhanced, near time zero signal, in the sense that it produces transitions in the parent molecule itself, not simply on the free fragments. Only then can it leave its imprint so strongly on the anisotropy of the fragment ion distribution. We believe that the resulting 3D angular distribution is in this case the convolution of a parallel transition induced by the 267 $\mathrm{nm}$ with another parallel transition induced by the $802 \mathrm{~nm}$ beam. This is why it still retains the anisotropy related to the polarization of the $267 \mathrm{~nm}$ beam, but since the polarization of the $802 \mathrm{~nm}$ beam points toward the detector, it causes an apparently unstructured velocity distribution of methyl fragments on the plane of the detector.

Further insight into the nature of the process can be gained by measuring power dependences as a function of the fluence of each of the laser pulses. For a long pump-probe delay time, it was confirmed that for both I and I $^{*}$ channels the signal intensities grow linearly with respect to the 267 $\mathrm{nm}$ power, but show a power dependence of sixth and fourth orders, respectively, with respect to $802 \mathrm{~nm}$. This confirms the idea that a highly nonlinear absorption leading to ionization is taking place in the methyl fragment after $A$-band dissociation, providing a certain degree of saturation that is common for a multiphoton absorption process. It can be argued that IR ionization of methyl is a highly nonlinear process, and therefore it is likely that Stark shifts in the energy levels do take place; however, since the arrival of the $802 \mathrm{~nm}$ pulse in the asymptotic case occurs well after $\mathrm{CH}_{3} \mathrm{I}$ dissociation has occurred, there is no distortion of the $A$-band dissociation process; the subsequent ionization of $\mathrm{CH}_{3}$ occurs nonresonantly, so that the experiment would not be sensitive to the presence of Stark shifts in the parent molecule. We then performed the same power dependence studies at delay times of $\sim 85 \mathrm{fs}$ (maximum $\mathrm{CH}_{3}{ }^{+}$enhancement). The main result was that the power law with respect to the $267 \mathrm{~nm}$ laser yields a value of $2.3 \pm 0.2$, and the measurements with respect to the $802 \mathrm{~nm}$ fluence showed an apparently linear behavior with a slope of $1.4 \pm 0.2$. This unexpectedly low value for the IR laser power dependence at zero delay time, together with the increase in the $267 \mathrm{~nm}$ slope, is a strong indication that a process of multiphoton nature for the UV laser pulse is taking place near temporal overlap.

\section{Velocity map imaging of the $\mathrm{CD}_{3}$ fragment}

The possibility that molecular Rydberg states could be playing an important role on the photofragmentation process led us to perform analogous measurements to those described above but for the $\mathrm{CD}_{3} \mathrm{I}$ isotopic variant. Femtosecond

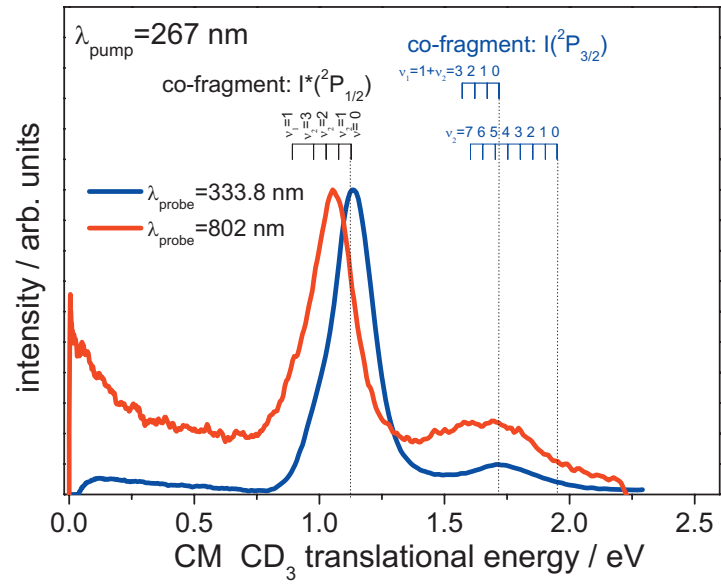

FIG. 10. Asymptotic (long delay time of $1 \mathrm{ps}$ ) $\mathrm{CM} \mathrm{CD}_{3}$ KEDs obtained at 802 (red) and $333.5 \mathrm{~nm}$ (blue) probe wavelengths from the $267 \mathrm{~nm}$ photodissociation of $\mathrm{CD}_{3} \mathrm{I}$ together with the assignments made for the different product channels. The curves have been normalized for clarity of the comparison.

photodissociation studies of the Rydberg states of $\mathrm{CH}_{3} \mathrm{I} / \mathrm{CD}_{3} \mathrm{I}$ have been performed in the past. ${ }^{17,23}$ Their lifetimes are known to exhibit an important isotope effect in dissociation since they are bound states with a C-I dissociation coordinate, which is coupled to the methyl umbrella mode. In $\mathrm{CD}_{3} \mathrm{I}$, Rydberg state lifetimes are known to increase in some cases up to a factor of 4 with respect to $\mathrm{CH}_{3} \mathrm{I} .{ }^{17}$

Figure 10 shows a comparison between the KEDs obtained at a long delay time in a nonresonant MPI (802 nm) probe experiment and that measured in an experiment with REMPI detection of $\mathrm{CD}_{3}$ at $333.8 \mathrm{~nm}$ [ $Q$ branch of the $3 p_{z}\left({ }^{2} A_{2}^{\prime \prime} \leftarrow{ }^{2} A_{2}^{\prime \prime}\right) 0_{0}^{0}$ transition]. As for $\mathrm{CH}_{3} \mathrm{I}$ [see Fig. 3(c)], in the second case the probe laser resonantly ionizes vibrationless $\mathrm{CD}_{3}(\nu=0)$. It is well known that in the photodissociation of $\mathrm{CD}_{3} \mathrm{I}$ at $266 \mathrm{~nm}$ the population of $\mathrm{CD}_{3}(\nu=0)$ in correlation with ground state iodine is practically negligible. ${ }^{24}$ However, we do observe two peaks in the distribution. The peak at lower kinetic energy corresponds to the $\mathrm{CD}_{3}(\nu=0)$ $+I^{*}\left({ }^{2} P_{1 / 2}\right)$ channel, but the higher energy peak appears at a kinetic energy well below that corresponding to the $\mathrm{CD}_{3}(\nu$ $=0)+\mathrm{I}\left({ }^{2} P_{3 / 2}\right)$ channel. We can assign this weak, broad peak to the channel yielding $\mathrm{CD}_{3}\left(\nu_{1}=1\right)$ in correlation with ground state iodine. As in the case of $\mathrm{CH}_{3}$, we can ionize $\mathrm{CD}_{3}$ in $\nu_{1}=1$ due to the overlap of the $Q$ branch of the $1^{1}{ }_{1}$ transition with the bandwidth of the probe laser, which is centered at the $Q$ branch of the $0^{0}{ }_{0}$ transition. This distribution contrasts with that obtained when using the nonresonant MPI probe at $802 \mathrm{~nm}$. As can be seen, in that case two broad peaks are observed, which are shifted to lower kinetic energies with respect to those obtained in the resonant experiment. In this case, we nonselectively multiphoton ionize all $\mathrm{CD}_{3}$ fragments regardless of their vibrational state and several $\nu_{2}$ states are observed (the peaks are broader). Actually, the peak at lower energy and specially that at higher energy are now significantly shifted to lower kinetic energies. Li and Hwang $^{24}$ measured the inverted vibrational distributions for both I and I* channels, peaking at $\nu_{2}=1$ for $\mathrm{CD}_{3}$ correlating with $\mathrm{I}^{*}$ and at $\nu_{2}=3-4$ for $\mathrm{CD}_{3}$ correlating with $\mathrm{I}^{24}$ The 

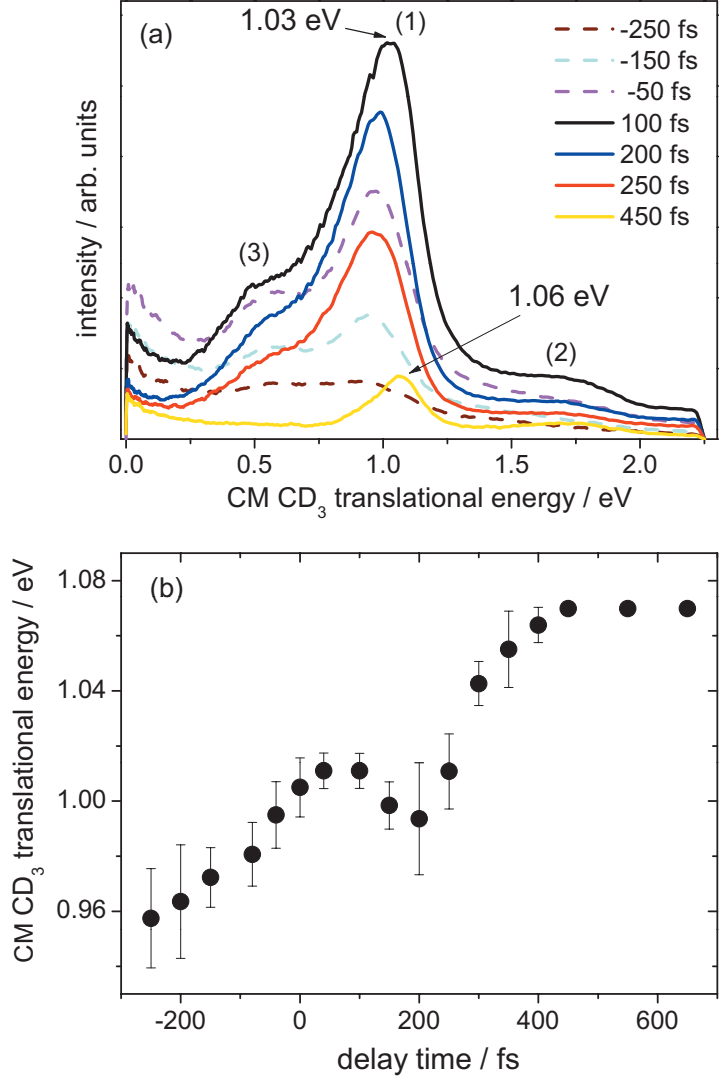

FIG. 11. (a) Same as in Fig. 6 but for $\mathrm{CD}_{3} \mathrm{I}$. (b) Same as in Fig. 7 but for $\mathrm{CD}_{3} \mathrm{I}$.

present results are in agreement with the large $\nu_{2}$ vibrational excitation of the $\mathrm{CD}_{3}$ fragment in the $266 \mathrm{~nm}$ photodissociation of $\mathrm{CD}_{3} \mathrm{I}$.

In Fig. 11 the behavior of the $\mathrm{CM} \mathrm{CD}_{3}$ KED at different delay times between the 267 and $802 \mathrm{~nm}$ pump-probe pulses [panel (a)] and the progressive shift in the position of the maximum of the peak labeled (1) in the KED as we move toward the asymptotic case [panel (b)] are depicted. In all respects, it is clear that the overall behavior is quite similar to that of $\mathrm{CH}_{3} \mathrm{I}$. The intermediate distributions in panel (a), taken close to the temporal overlap between the pump and probe pulses, also show the presence of what appear to be the same two asymptotic channels, except shifted toward lower energies and broadened in energy. As in the $\mathrm{CH}_{3} \mathrm{I}$ case, the shift toward lower energy values diminishes as the delay time is increased, until it disappears around $+400 \mathrm{fs}$, where the asymptotic situation is recovered.

The transients obtained for the three peaks [labeled (1), (2), and (3) in Fig. 11(a)] are quite similar to those observed for $\mathrm{CH}_{3} \mathrm{I}$ (Fig. 5). The conclusion is that no detectable isotope effect is observed either in the parent ion temporal behavior or in the $\mathrm{CH}_{3} / \mathrm{CD}_{3}$ fragment energies, dynamics, or angular distributions.

\section{Velocity map imaging of the I fragment}

We have measured velocity map ion images for $\mathrm{I}^{+}$. As in the $\mathrm{CH}_{3} / \mathrm{CD}_{3}$ case, a similar enhanced signal is found around zero delay time. However, no clear ring structure is observed in the enhanced $\mathrm{I}^{+}$images, but just a broad structureless MPI.
At long delay times (asymptotic region), a small peak centered at $0.14 \mathrm{eV}$ is observed in the KED over a large background, which corresponds to the $\mathrm{CH}_{3}+\mathrm{I}^{*}\left({ }^{2} P_{1 / 2}\right)$ channel of the $A$-band photodissociation of $\mathrm{CH}_{3} \mathrm{I}$ at $267 \mathrm{~nm}$ (not shown). No trace of the $\mathrm{CH}_{3}+\mathrm{I}\left({ }^{2} P_{3 / 2}\right)$ channel is observed. As delay time decreases, a very broad structureless signal centered at $\sim 0.10 \mathrm{eV}$ starts to evolve reaching a maximum at around time zero and then decreasing. Thus, although we do see an enhancement of the signal as we approach time zero, we attribute this enhancement to a different multiphoton process when compared to $\mathrm{CH}_{3} / \mathrm{CD}_{3}$.

\section{DISCUSSION}

It is the aim of this section to propose a mechanism for the dynamic behavior reported for the dissociative process, mainly concentrating on the formation of $\mathrm{CH}_{3}{ }^{+}$. We will show how, according to this mechanism, as the molecular wave packet evolves in the repulsive surfaces of the $A$-band, we can observe a smooth transition between the situation that leads to the formation of the parent ion to that leading to the formation of fragment ions, thus explaining the temporal shift in the signals observed for the parent and the fragment ions (Figs. 2 and 5) and the rest of experimental features. In the first part of this discussion, we will present a summary of other possible descriptions, involving ion and Rydberg state dynamics, that we believe show profound incompatibilities with the observations. Later, we will provide a detailed account of the proposed mechanism, supported by the experimental data, where wave packet evolution on the $A$-band opens and closes ionization channels that yield completely different products of the reaction.

We will first consider the possibility that the transient behaviors observed are a reflection of ion dynamics. Within this category, we will distinguish between dissociative ionization upon simultaneous pump-probe absorption (option 1), or two-step processes, where either the ion is initially pumped into a dissociative or predissociative state (option 2) or it is formed in a bound state, but later suffers a further promotion into a dissociative state (option 3). In order to follow the discussion in terms of possible ion dynamics, it is interesting to consider the energetics of the different ionic channels that could be involved. ${ }^{25}$ The ground state of $\mathrm{CH}_{3} \mathrm{I}^{+}\left(X^{2} E_{1 / 2,3 / 2}\right)$ correlates with ion and neutral fragments as follows:

$$
\mathrm{CH}_{3} \mathrm{I}^{+}\left(\tilde{X}^{2} E_{3 / 2,1 / 2}\right) \rightarrow \mathrm{CH}_{3}{ }^{+}+\mathrm{I}\left({ }^{2} P_{3 / 2}\right) .
$$

In addition, the first excited state of $\mathrm{CH}_{3} \mathrm{I}^{+}(\widetilde{A})$ correlates with $\mathrm{I}^{+}$and neutral $\mathrm{CH}_{3}$ as

$$
\mathrm{CH}_{3} \mathrm{I}^{+}(\widetilde{A}) \rightarrow \mathrm{CH}_{3}+\mathrm{I}^{+}\left({ }^{3} P_{2}\right) \text {. }
$$

Both the ground and first excited states of $\mathrm{CH}_{3} \mathrm{I}^{+}$are bound and it is known that the $\widetilde{A}$ state can internally convert to the ground state. The $\widetilde{B}$ state of $\mathrm{CH}_{3} \mathrm{I}^{+}$should have a moderate spin-orbit splitting with the same symmetry as the ionic ground state. No spectroscopic structure has been found that can be attributed to the $\widetilde{B}$ state and thus, given the small energy difference between the $\widetilde{A}$ and $\widetilde{B}$ states, it could be 


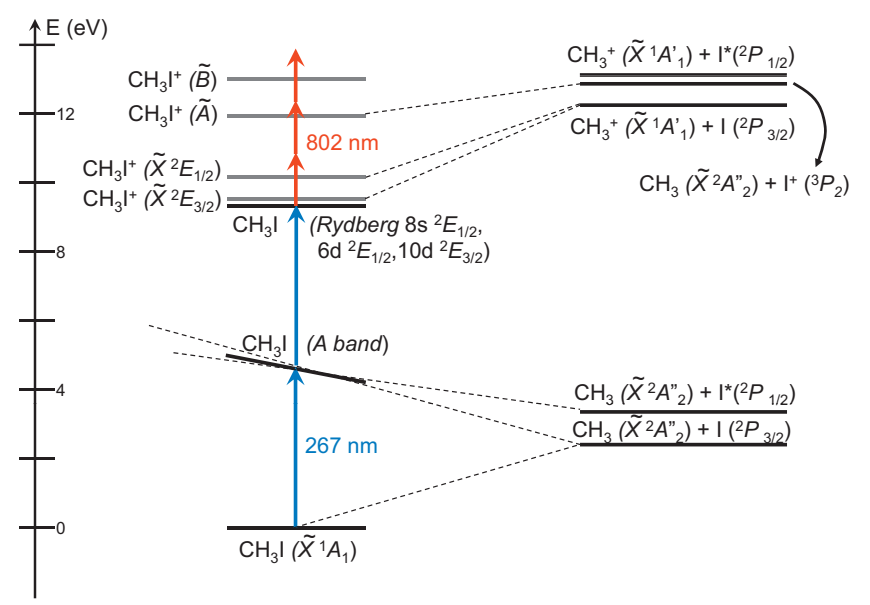

FIG. 12. Schematic representation of the energy levels of neutral and ionic $\mathrm{CH}_{3} \mathrm{I}$ parent molecule and fragments. Energy levels for the ionic channels taken from Ref. 25. The arrows indicate multiple 267 and $802 \mathrm{~nm}$ photons absorbed to reach the ionic ladder of $\mathrm{CH}_{3} \mathrm{I}$.

possible that the $\widetilde{B}$ state suffers a fast internal conversion to the $\widetilde{A}$ state or it is crossed by a dissociative state, in any case, undergoing dissociation. ${ }^{25}$ A diagram showing the known energy levels is depicted in Fig. 12.

The first option (dissociative ionization) would correspond to a scenario where simultaneous absorption of 267 and $802 \mathrm{~nm}$ pulses would take place to reach an ion state of sufficiently high energy so as to yield fragment ions; that is, ion dissociation would produce $\mathrm{CH}_{3}{ }^{+} / \mathrm{CD}_{3}{ }^{+}$directly. There are several drawbacks in this picture to explain our observations. First, it would be hard to explain the temporal shift between the fragment signals and the parent signals, since without subsequent dynamics, it would be expected that all species would peak simultaneously (as it is the case for the low kinetic energy channel at $\sim 0.6 \mathrm{eV}$; see Figs. 5 and 6). Second, this picture would be at a loss to explain the smooth shift in the KED peak values observed both for $\mathrm{CH}_{3}{ }^{+}$(Fig. 6) and $\mathrm{CD}_{3}{ }^{+}$(Fig. 11). Instead, we would expect a rather unstructured KED near temporal overlap, followed by a change into the completely different distribution at long delay times (actually, this is what we observe for $\mathrm{I}^{+}$). Third, the enhanced, structured distribution of $\mathrm{CH}_{3} / \mathrm{CD}_{3}$ ions is absent upon irradiation with either the 267 or the $802 \mathrm{~nm}$ pulses separately, even though a broad range of intensities was explored; one would expect that at least with the $802 \mathrm{~nm}$ pulse alone, it should be possible to reach the same states through a highly multiphoton process if sufficiently high intensities are used. And finally, upon a multiphoton multicolor absorption, the electron would carry all of the excess kinetic energy, leaving the cation with little available energy. The $\mathrm{CH}_{3} \mathrm{I}^{+}$ does not have dissociative states in the first few eV (see Fig. 12). ${ }^{25}$ If the transition takes place to one of the bound states, it would be expected that it would remain bound, contrary to the observation of $\mathrm{CH}_{3}{ }^{+}$.

One could argue another type of ionic dynamics (option 2): One where population could be transferred first to a dissociative or predissociative ionic state of the molecule by the pump laser and then promoted into a higher lying $\mathrm{CH}_{3} \mathrm{I}^{+}$ dissociative state by the probe laser from where, in turn, the parent ion would dissociate into the ionic fragments observed. It is well known that the ground state of $\mathrm{CH}_{3} \mathrm{I}^{+}$is bound and long lived since many of its spectroscopic properties have been thoroughly studied with high sensitivity and resolution: spin-orbit splitting, vibrational level constants, etc. ${ }^{25}$ The first ionic excited state $\mathrm{CH}_{3} \mathrm{I}^{+}(\widetilde{A})$ is bound but predissociates around the threshold to the formation of $\mathrm{CH}_{3}{ }^{+}$ and thus the nascent methyl ions originating through this process would carry very low kinetic energy; moreover, this $\widetilde{A}$ state is known to have a relatively long lifetime of at least several picoseconds, which is inconsistent with the fast enhancement signals. Higher states are known to be unbound and quickly dissociative. The probability of absorption is lower for them due to the need for a higher order nonlinear process. It seems unlikely that in a situation where such processes were important, we would only see contribution from clearly distinguishable channels, as opposed to a combination of multiple channels.

Finally, a different type of ion dynamics could be possible (option 3). In it, the ion could be formed in a bound state in the first step (by absorption of several photons of 267 $\mathrm{nm}$ ), from where a standard pump-probe process would follow. The UV pulse would then be acting both as a pure ionizer and as the pump in a process taking place in the $\mathrm{CH}_{3} \mathrm{I}^{+}$molecular ion. ${ }^{26,27}$ In this case, the ion dissociation could lead to neutral $\mathrm{CH}_{3}$, which would be later ionized by the probe laser. This type of mechanism could explain different temporal transients for the different species, but it is still incompatible with the experiment mainly because the lowest ion state in correlation with the formation of neutral $\mathrm{CH}_{3}$ also correlates to $\mathrm{I}^{+}$, not to $\mathrm{I} / \mathrm{I}^{*}$, so the appearance of two channels would be unaccounted for. Additionally, it would involve the absorption of a very large number of photons, both from the pump and probe beams, in contradiction with the measured power dependences, even if some degree of saturation takes place.

In summary, we believe that none of the descriptions involving dynamics on the ion surfaces can account for the experimental findings described here. Thus, we conclude that the observation of this enhanced contribution to the $\mathrm{CH}_{3}{ }^{+}$ signal must be related to neutral photodissociation dynamics of an excited state in the $\mathrm{CH}_{3} \mathrm{I}$ molecule. Mainly, the possibilities are reduced either to the $A$-band or to Rydberg states. It is true that the power dependence at short delay time indicates multiphoton absorption of the UV light. As it was mentioned before, by absorption of two pump photons (9.275 eV with a bandwidth of around $0.05 \mathrm{eV}$ ), two-photon excitation is possible to several high-lying members of Rydberg series, ${ }^{18}$ namely, $10 p{ }^{2} E_{3 / 2}, 9 d^{2} E_{3 / 2}, 10 d^{2} E_{3 / 2}, 8 s^{2} E_{1 / 2}$, and $6 d^{2} E_{1 / 2}$. From those Rydberg states, at least two $802 \mathrm{~nm}$ photons would be needed to reach an ion state sufficiently high lying so that fragment ion formation is possible. This process may be able to explain the temporal shifts observed between the parent and fragment ions, but using the known energetics, ${ }^{25}$ there is no combination of pump and probe laser pulses related to dissociation of a Rydberg state that can yield methyl fragments with kinetic energies near the values observed experimentally; that is, similar to those of the neu- 


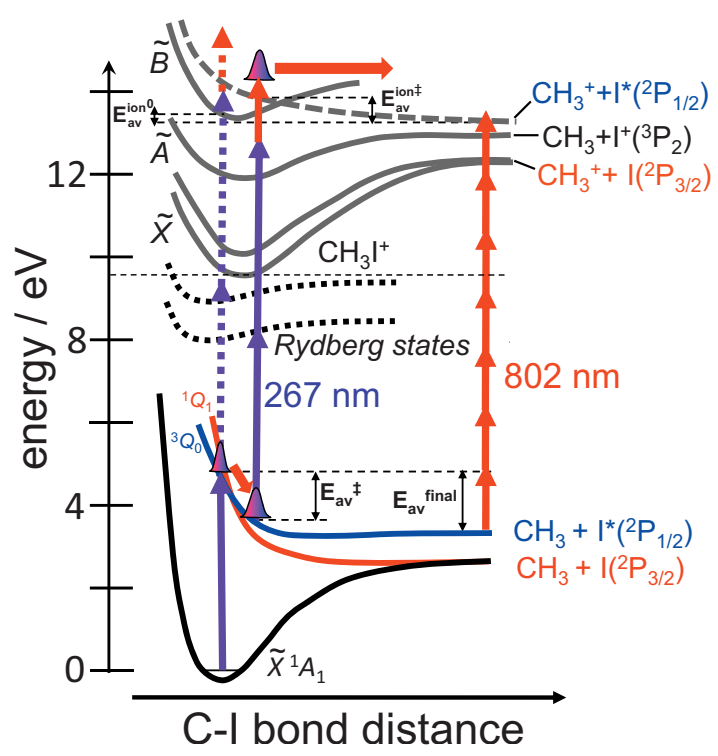

FIG. 13. Schematic representation of the proposed mechanism for the detection of transient species and final products in the real time photodissociation of $\mathrm{CH}_{3} \mathrm{I}$ in the $A$-band by multiple absorption of pump and probe laser photons. See the text for details.

tral channels arising from the $A$-band dissociation. Moreover, angular anisotropies from these highly excited states would have to match those of the $A$-band dissociation. This, together with the $\mathrm{CD}_{3} \mathrm{I}$ experiments yielding very short lifetimes, excludes the possibility of photodissociation on highlying Rydberg states as responsible for the observations.

We could also consider the possibility that lower Rydberg states may be participating in the process; that is, either the $B$-band at around $6.2 \mathrm{eV}$, accessible with one $267 \mathrm{~nm}$ photon and one $802 \mathrm{~nm}$ photon, or even the $C$-band, for which one $267 \mathrm{~nm}$ photon and two $802 \mathrm{~nm}$ photons would be required. The $B$-band is well known for $\mathrm{CH}_{3} \mathrm{I}$ with vibronic bands assigned. ${ }^{23}$ The lifetimes of the Rydberg states are considerably long (of the order of picoseconds), given that they are bound and only dissociate through coupling with the $4 E$ or ${ }^{2} A_{2}$ repulsive states correlating with $\mathrm{CH}_{3}+\mathrm{I}^{*}\left({ }^{2} P_{1 / 2}\right){ }^{9,28}$ For comparison, we have performed experiments studying the femtosecond $B$-band photodissociation with a pump laser centered at $\sim 200 \mathrm{~nm},{ }^{29}$ and the results have shown radically different features in terms of temporal behavior (of the order of picoseconds typical of an electronic predissociation process), KEDs (only the I* channel can be seen), and anisotropy. The $C$-band is less well known, but a behavior not completely dissimilar from the $B$-band would be expected. Participation of the Rydberg states, therefore, cannot account for the observed results.

Having discarded the ion or Rydberg dynamics as responsible for the experimental results, we believe that the strongly enhanced signal obtained at early delay times showing a global structure with strong similarities to the asymptotic case reflects early-time dynamics of the molecular system on the $A$-band. For such short times, we propose a mechanism where $\mathrm{CH}_{3} \mathrm{I}$ absorbs a $267 \mathrm{~nm}$ photon in the early part of the pulse and initiates $A$-band dissociation. This mechanism is schematically depicted in Fig. 13. In a fraction of molecules, the wave packet will evolve undisturbed and give rise to the neutral fragments $\mathrm{CH}_{3}$ and I, which are amenable to be probed by a delayed $802 \mathrm{~nm}$ pulse at long delay times (asymptotic component). In part of the $A$-band excited molecules, however, subsequent absorption of additional 267 $\mathrm{nm}$ photons is possible; in fact it is likely to be favored by the presence of high-lying Rydberg states just below the ionization continuum, and only one additional $802 \mathrm{~nm}$ photon is enough to produce $\mathrm{CH}_{3} \mathrm{I}^{+}$in its ground state. This $2+1^{\prime}$ process for the formation of the molecular ion $\mathrm{CH}_{3} \mathrm{I}^{+}$would have a strong probability due to the resonant enhancement for the $267 \mathrm{~nm}$ photons at one- and two-photon level, and the strong absorption probability of the $802 \mathrm{~nm}$, which would produce the ion with little excess energy above the ionization potential.

Given the very rapid dissociation in the $A$-band $(\approx 100$ fs), ${ }^{4,7,8}$ the transient species $\left[\mathrm{CH}_{3} \cdots \mathrm{I}\right]^{*}$ can still absorb further $267 \mathrm{~nm}$ photons coming from the trailing edge of the pulse even for considerably elongated internuclear distances. If sufficient time has passed before absorption takes place, the wave packet on the $A$-band will have already split due to the early nonadiabatic crossing between the ${ }^{3} Q_{0}$ and ${ }^{1} Q_{1}$ surfaces, acquiring different excess kinetic energies. Upon simultaneous 267 and $802 \mathrm{~nm}$ absorption, it would be expected that the part of the wave packet evolving on the ${ }^{1} Q_{1}$ surface could be promoted to the $\tilde{A}$ excited state of $\mathrm{CH}_{3} \mathrm{I}^{+}$, which is known to undergo a fast internal conversion to the $\mathrm{CH}_{3}{ }^{+}+\mathrm{I}\left({ }^{2} P_{3 / 2}\right)$ asymptote (ground state of the ion), and the part evolving on ${ }^{3} Q_{0}$ to the $\widetilde{B}$ state, which would dissociate to the $\mathrm{CH}_{3}{ }^{+}+\mathrm{I}^{*}\left({ }^{2} P_{1 / 2}\right)$ asymptote (see Fig. 13). The $\tilde{A}$ state of the ion is a bound state, but there is less information about the $\widetilde{B}$ state, which is supposed to yield dissociation most probably by crossing with a dissociative potential surface. ${ }^{25}$ In our mechanism, when absorption of additional pump and probe pulses takes place from the ${ }^{3} Q_{0}$ surface when the C-I internuclear distance has elongated considerably (so that the fragments have already acquired some kinetic energy), the transition would be to a repulsive potential surface, and therefore the loss of an electron would be followed by dissociation into $\mathrm{CH}_{3}{ }^{+}+\mathrm{I}^{*}\left({ }^{2} P_{1 / 2}\right)$. In the case of the wave packet evolving on the ${ }^{1} Q_{1}$ potential surface, the absorption would be to the $\tilde{A}$ state of the ion, which then internally converts to the $\mathrm{CH}_{3}{ }^{+}+\mathrm{I}\left({ }^{2} P_{3 / 2}\right)$ products.

Considering this mechanism, which is very much in the spirit of the methodology presented by Zhong and Zewail in Ref. 4, the kinetic energy finally present in the methyl ion fragment should contain two contributions. The first contribution arises from the available energy of the neutral $A$-band dissociation at a given intermediate $\mathrm{C}-\mathrm{I}$ internuclear distance $\left(E_{\mathrm{av}}{ }^{\ddagger}\right)$, which will be smaller than the asymptotic available energy $\left(E_{\mathrm{av}}{ }^{\text {final }}\right)$, since the wave packet cannot have reached a very long internuclear distance at the time of the second absorption (see Fig. 13). Since the excess energy upon the ionization step will be taken by the ejected electron, the other contribution comes only from the available energy on the ionic repulsive potential surface once the wave packet has initially evolved in the neutral potential surface. The $\mathrm{CH}_{3} / \mathrm{CD}_{3}$ kinetic energy shifts observed for channels $\mathrm{CH}_{3}$ $+\mathrm{I}^{*}$ for $\mathrm{CH}_{3} \mathrm{I}$ (Fig. 7) and $\mathrm{CD}_{3}+\mathrm{I}^{*}$ for $\mathrm{CD}_{3} \mathrm{I}$ [Fig. 11(b)] 
indicate that the methyl fragment carries less energy close to the temporal overlap of the pump and probe pulses than in the asymptotic region (long delay time), which can be explained if the ionic dissociative surface is flatter than the neutral surface. The fact that we observe a larger shift as the delay time is shortened is an indication that the $802 \mathrm{~nm}$ pulse provides the time when the wave packet is frozen in the neutral dissociation surface and taken to the flatter ionic dissociative surface where the total energy gained is bound to be lower. The broadened KED peaks measured for short delay times are also consistent with this mechanism, where excitation is produced in a region of the neutral dissociative surface of rapidly changing potential, and therefore, parts of the wave packet with a range of kinetic energies will contribute for a given delay time. The shape of the curves shown in Figs. 7 and 11(b) directly reflects the shapes of the neutral and ionic potential energy surfaces involved in the overall process.

This description would also account for the delay time observed in the $\mathrm{CH}_{3}{ }^{+}$transients with respect to that of $\mathrm{CH}_{3} \mathrm{I}^{+}$ (see Fig. 5). Initially, the wave packet will have acquired a negligible kinetic energy on the neutral repulsive surfaces (A-band). As depicted in Fig. 13, upon absorption to a bound ionic excited state, the wave packet will not posses enough energy to escape the potential well. It is only when the wave packet in the neutral surfaces has evolved far enough that the ion will be formed in a state with a total energy above the dissociation asymptote.

The proposed mechanism provides an adequate description to all the experimental observations. We can explain the pump and probe power dependences and anisotropies measured at long delay times and close to time zero, since multiple 267 and $802 \mathrm{~nm}$ photon absorption is involved depending on the delay time regime (ionization of transient species or free fragments). The time delay between the parent ion transient and that of the $\mathrm{CH}_{3}$ fragment also agrees with the proposed mechanism since the maximum enhancement of the $\mathrm{CH}_{3}{ }^{+}$signal occurs when the $A$-band dissociation has proceeded to some extent. The present model also explains the absence of isotopic effect and the effect of changing the polarization of the probe laser, since the $802 \mathrm{~nm}$ pulse behaves also as pump pulse with respect to the transient species. Finally, the model is compatible with the increasing kinetic energies observed for $\mathrm{CH}_{3}{ }^{+}$as delay time increases up to the asymptotic case.

\section{SUMMARY}

In the present work we have studied the femtosecond photodissociation dynamics of $\mathrm{CH}_{3} \mathrm{I}$ and $\mathrm{CD}_{3} \mathrm{I}$ in the $A$-band using pump and probe photons centered at 267 and $802 \mathrm{~nm}$, respectively. The IR probe pulse is used to nonresonantly ionize the parent molecule and final products and $\mathrm{CH}_{3}{ }^{+} / \mathrm{CD}_{3}{ }^{+}$and $\mathrm{I}^{+}$images are measured under velocity map conditions. The measured $\mathrm{CH}_{3}{ }^{+} / \mathrm{CD}_{3}{ }^{+}$images at delay times close to zero are characterized by enhanced features which resemble those obtained at long delay times (asymptotic conditions). Experimental data include KEDs and anisotropies, alignment dynamics, and polarization and isotopic effects. A detailed discussion has been carried out to discard the possibility of ion or Rydberg state dynamics to explain the experimental measurements. A mechanism based on the combination of different multiple absorption of 267 and $800 \mathrm{~nm}$ photons depending on the configuration of the C-I bond is proposed, which agrees with the experimental observations. This work demonstrates that the use of an IR nonresonant MPI scheme in combination with UV excitation radiation and the velocity map imaging technique are well suited to study the real time photodissociation dynamics of transient species and final products of polyatomic molecules. Work is in progress to extend this strategy to other complex molecular systems.

\section{ACKNOWLEDGMENTS}

We thank Professor T. Baumert and Professor M. Wollenhaupt for helpful discussions. J.D. gratefully acknowledges financial support through a fellowship of the Unidad Asociada between Universidad Complutense de Madrid (UCM) and CSIC. This work has been financed by the Spanish MCINN through Grant No. CTQ2008-02578/BQU and Consolider program SAUUL through Grant No. CSD200700013 and by European Comission through ITN Program FASTQUAST (PITN-GA-2008-214962). This research has been carried out within the Unidad Asociada "Química Física Molecular" between Departamento de Química Física I of UCM and CSIC. The facilities provided by the Centro de Asistencia a la Investigación de Espectroscopia Multifotónica y de Femtosegundo (UCM) are gratefully acknowledged.

${ }^{1}$ J. C. Polanyi and A. H. Zewail, Acc. Chem. Res. 28, 119 (1995).

${ }^{2}$ M. Dantus, M. J. Rosker, and A. H. Zewail, J. Chem. Phys. 89, 6128 (1988).

${ }^{3}$ T. Baumert, S. Pedersen, and A. H. Zewail, J. Phys. Chem. 97, 12447 (1993).

${ }^{4}$ D. Zhong and A. H. Zewail, J. Phys. Chem. A 102, 4031 (1998).

${ }^{5}$ B. J. Greenblatt, M. T. Zanni, and D. M. Neumark, Science 276, 1675 (1997).

${ }^{6}$ D. Strasser, F. Goulay, and S. R. Leone, J. Chem. Phys. 127, 184305 (2007).

${ }^{7}$ R. de Nalda, J. G. Izquierdo, J. Durá, and L. Bañares, J. Chem. Phys. 126, 021101 (2007).

${ }^{8}$ R. de Nalda, J. Durá, A. García-Vela, J. G. Izquierdo, J. GonzálezVázquez, and L. Bañares, J. Chem. Phys. 128, 244309 (2008).

${ }^{9}$ A. B. Alekseyev, H.-P. Liebermann, R. J. Buenker, and S. N. Yurchenko, J. Chem. Phys. 126, 234102 (2007).

${ }^{10}$ T. F. Hunter and K. S. Kristjansson, Chem. Phys. Lett. 58, 291 (1978).

${ }^{11}$ R. S. Mulliken, J. Chem. Phys. 8, 382 (1940).

${ }^{12}$ A. T. J. B. Eppink and D. H. Parker, J. Chem. Phys. 109, 4758 (1998).

${ }^{13}$ J. Durá, R. de Nalda, J. Álvarez, J. G. Izquierdo, G. A. Amaral, and L. Bañares, ChemPhysChem 9, 1245 (2008).

${ }^{14}$ Our design follows the original design by Professor D. Gerlich that can be found in the web page http://www.tu-chemnitz.de/physik/ion/ technology/piezo_valve/index.html.

${ }^{15}$ A. T. Eppink and D. H. Parker, Rev. Sci. Instrum. 68, 3477 (1997).

${ }^{16}$ G. A. García, L. Nahon, and I. Powis, Rev. Sci. Instrum. 75, 4989 (2004).

${ }^{17}$ M. H. M. Janssen, M. Dantus, H. Guo, and A. H. Zewail, Chem. Phys. Lett. 214, 281 (1993).

${ }^{18}$ S. Eden, P. Limao-Vieira, S. V. Hoffmann, and N. J. Mason, Chem. Phys. 331, 232 (2007).

${ }^{19}$ G. Li, H. J. Hwang, and H. C. Jung, Rev. Sci. Instrum. 76, 023105 (2005).

${ }^{20}$ A. T. J. B. Eppink and D. H. Parker, J. Chem. Phys. 110, 832 (1999). 
${ }^{21}$ E. Hamilton, T. Seideman, T. Ejdrup, M. D. Poulsen, C. Z. Bisgaard, S. Viftrup, and H. Stapelfeldt, Phys. Rev. A 72, 043402 (2005).

${ }^{22}$ H. Stapelfeldt and T. Seideman, Rev. Mod. Phys. 75, 543 (2003).

${ }^{23}$ A. P. Baronavski and J. C. Owrutsky, J. Chem. Phys. 108, 3445 (1998).

${ }^{24}$ G. Li and H. J. Hwang, J. Chem. Phys. 124, 244306 (2006).

${ }^{25}$ K. Walter, R. Weinkauf, U. Boesl, and E. W. Schlag, J. Chem. Phys. 89, 1914 (1988)
${ }^{26}$ F. Aguirre and S. T. Pratt, J. Chem. Phys. 122, 234303 (2005).

${ }^{27}$ F. Wang, M. L. Lipciuc, X. M. Yang, and T. N. Kitsopoulos, Phys. Chem. Chem. Phys. 11, 2234 (2009).

${ }^{28}$ D. J. Donaldson, M. S. Child, and V. Vaida, J. Chem. Phys. 88, 7410 (1988).

${ }^{29}$ G. Gitzinger, M. E. Corrales, G. A. Amaral, J. Durá, R. de Nalda, and L. Bañares (in preparation). 